Article

\title{
Syzygies, Betti Numbers, and Regularity of Cover Ideals of Certain Multipartite Graphs
}

\author{
A. V. Jayanthan ${ }^{1, *}$ and Neeraj Kumar ${ }^{2}$ \\ 1 Department of Mathematics, Indian Institute of Technology Madras, Chennai 600036, India \\ 2 Department of Mathematics, Indian Institute of Technology Hyderabad, Kandi, Sangareddy 502285, India; \\ neeraj@iith.ac.in or neeraj.unix@gmail.com \\ * Correspondence: jayanav@iitm.ac.in
}

Received: 10 August 2019; Accepted: 13 September 2019; Published: 19 September 2019

check for updates

\begin{abstract}
Let $G$ be a finite simple graph on $n$ vertices. Let $J_{G} \subset K\left[x_{1}, \ldots, x_{n}\right]$ be the cover ideal of $G$. In this article, we obtain syzygies, Betti numbers, and Castelnuovo-Mumford regularity of $J_{G}^{s}$ for all $s \geq 1$ for certain classes of graphs $G$.
\end{abstract}

Keywords: syzygy; Betti number; Castelnuovo-Mumford regularity; bipartite graph; multipartite graph

\section{Introduction}

In this article, we compute the resolution, syzygies, and Betti numbers of powers of certain classes of squarefree monomial ideals. As a by-product, we obtain the expression for the regularity of powers of these classes of ideals. Computation of minimal free resolution and syzygies of ideals and modules over polynomial rings have always attracted researchers in commutative algebra and algebraic geometry. Recently, there has been much interest in studying the homological aspects of squarefree monomial ideals in polynomial rings. As these ideals have strong combinatorial connections, problems in this area have attracted both commutative algebrists and combinatorists. Even in this case, there are only very few cases of ideals for which explicit computation of the resolution, including the complete description of syzygies, is done. The main open problems in this area are to find/construct minimal free resolutions in more cases, and to introduce new ideas and structures ([1] Remark 2.6). There are even less results on the resolution of powers of ideals. If $I$ is generated by a regular sequence, then $I^{s}$ is a determinantal ideal and hence the minimal free resolution of $I^{s}$ can be obtained by using the Eagon-Northcott complex [2]. As the maps in this resolution may not be degree preserving, the computation of graded Betti numbers and more generally the computation of the syzygies may not be possible. In the work by the authors of [3], they explicitly compute the graded Betti numbers of $I^{s}$ if $I$ is a homogeneous complete intersection in a polynomial ring.

Among the resolutions, linear resolutions are possibly the simplest to describe. Let $R=$ $K\left[x_{1}, \ldots, x_{n}\right]$, where $K$ is a field. If $I$ is the defining ideal of the rational normal curve in $\mathbb{P}^{n-1}$, then Conca proved that $I^{s}$ has a linear resolution for all $s$ [4]. Herzog, Hibi, and Zheng proved that if $I$ is a monomial ideal generated in degree 2, then $I$ has linear resolution if and only if $I$ has linear quotients if and only if $I^{s}$ has a linear resolution for all $s \geq 1$ [5]. It was proved by Conca and Herzog [6], that if $I$ is a polymatroidal ideal in $R$, then $I$ has linear quotients. Moreover, they proved that the product of polymatroidal ideals are polymatroidal. Therefore, if $I$ is polymatroidal, then $I^{s}$ has linear resolution for all $s \geq 1$. If $I$ is a lexsegment ideal, then Ene and Olteanu proved that $I$ has linear resolution if and only if $I$ has linear quotients if and only if $I^{s}$ has linear resolution for all $s \geq 1$ if and only if $I^{s}$ has linear quotients for all $s \geq 1$ [7]. 
In all the above-mentioned results, the authors do not compute the syzygy modules. In general, it is a nontrivial task to compute the syzygy modules, even when the resolution is linear. It was shown by Kodiyalam [8], and independently by Cutkosky, Herzog, and Trung [9], that if $I$ is a homogeneous ideal in a polynomial ring, then there exist non-negative integers $d, e$ and $s_{0}$ such that $\operatorname{reg}\left(I^{s}\right)=d s+e$ for all $s \geq s_{0}$, where reg $(-)$ denote the Castelnuovo-Mumford regularity. Kodiyalam proved that $d \leq \operatorname{deg}(I)$, where $\operatorname{deg}(I)$ denotes the largest degree of a homogeneous minimal generator of $I$. In general, the stability index $s_{0}$ and the constant term $e$ are hard to compute. There have been discrete attempts in identifying $s_{0}$ and $e$ for certain classes of ideals.

Recently, there have been a lot of activity in studying the interplay between the combinatorial properties of graphs and the algebraic properties of ideals associated to graphs. For a finite simple graph $G$ on the vertex set $\left\{x_{1}, \ldots, x_{n}\right\}$, let $J_{G} \subset R=K\left[x_{1}, \ldots, x_{n}\right]$, where $K$ is a field, denote the cover ideal of $G$ (see Section 2 for the definition). It may be noted that $J_{G}=\cap_{\left\{x_{i}, x_{j}\right\} \in E(G)}\left(x_{i}, x_{j}\right)$ is the Alexander dual of the edge ideal of $I$ (see Section 2 for the definition). Although the connection between algebraic properties of the edge ideal and combinatorial properties of the graph has been studied extensively, not much is known about the connection between the properties of the cover ideal and the graph. In [10], Seyed Fakhari studied certain homological properties of symbolic powers of cover ideals of very well covered and bipartite graphs. It was shown that if $G$ is a very well covered graph and $J_{G}$ has a linear resolution, then $J_{G}^{(s)}$ has a linear resolution for all $s \geq 1$. Furthermore, it was proved that if $G$ is a bipartite graph with $n$ vertices, then for $s \geq 1$,

$$
\operatorname{reg}\left(J_{G}^{s}\right) \leq s \operatorname{deg}\left(J_{G}\right)+\operatorname{reg}\left(J_{G}\right)+1 .
$$

Hang and Trung [11] studied unimodular hypergraphs and proved that if $\mathcal{H}$ is a unimodular hypergraph on $n$ vertices and rank $r$ and $J_{\mathcal{H}}$ is the cover ideal of $\mathcal{H}$, then there exists a non-negative integer $e \leq \operatorname{dim}\left(R / J_{\mathcal{H}}\right)-\operatorname{deg}\left(J_{\mathcal{H}}\right)+1$, such that

$$
\operatorname{reg} J_{\mathcal{H}}^{s}=\operatorname{deg}\left(J_{\mathcal{H}}\right) s+e
$$

for all $s \geq \frac{r n}{2}+1$. Since bipartite graphs are unimodular, their results hold true in the case of bipartite graphs as well. While the first result gives an upper bound for the constant term, the later result gives the upper bound for both the stability index and the constant term.

In this article, we obtain the complete description of the minimal free resolution, including the syzygies, of $J_{G}^{s}$ for some classes of multipartite graphs. The paper is organized as follows. In Section 2, we collect the preliminaries required for the rest of the paper. We study the resolution of powers of cover ideals of certain bipartite graphs in Section 3. If $G$ is a complete bipartite graph, then $J_{G}$ is a regular sequence and hence the minimal graded free resolution of $J_{G}^{s}$ can be obtained from (Theorem 2.1 in the work by the authors of [3]). We then move on to study some classes of bipartite graphs which are not complete. We obtain the resolution, syzygies and Betti numbers of powers of cover ideals of certain bipartite graphs, and as a by-product, we obtain expression for the regularity of powers of these ideals. Section 4 is devoted to the study of resolution and regularity of powers of cover ideals of certain complete multipartite graphs.

The main results of this article are the following: When $G$ is the cycle of length three or the complete graph on 4 vertices, we describe the graded minimal free resolution of $J_{G}^{s}$ for all $s \geq 1$. This allow us to compute the Betti numbers, Hilbert series and the regularity of $J_{G}^{s}$ for all $s \geq 1$. As a consequence, for cover ideals of complete tripartite and 4-partite graphs, we obtain precise expressions for the Betti numbers and the regularity of $J_{G}^{s}$. We conclude our article with a conjecture on the resolution of $J_{G}^{s}$ for all $s \geq 1$, where $G$ is a complete multipartite graph.

\section{Preliminaries}

In this section, we set the notation for the rest of the paper. All the graphs that we consider in this article are finite, simple, and without isolated vertices. For a graph $G, V(G)$ denotes the set of all 
vertices of $G$ and $E(G)$ denotes the set of all edges of $G$. A graph $G$ is said to be a "complete multipartite" graph if $V(G)$ can be partitioned into sets $V_{1}, \ldots, V_{k}$ for some $k \geq 2$, such that $\{x, y\} \in E(G)$ if and only if $x \in V_{i}$ and $y \in V_{j}$ for $i \neq j$. When $k=2$, the graph is called a "complete bipartite" graph. If $k=2$ with $\left|V_{1}\right|=m$ and $\left|V_{2}\right|=n$, we denote the corresponding complete bipartite graph by $K_{m, n}$ or by $K_{V_{1}, V_{2}}$. If $G$ and $H$ are graphs, then $G \cup H$ denote the graph on the vertex set $V(G) \cup V(H)$ with $E(G \cup H)=E(G) \cup E(H)$. A graph $G$ is called a "bipartite" graph if $V(G)=V_{1} \sqcup V_{2}$ such that $\{x, y\} \in E(G)$ only if $x \in V_{1}$ and $y \in V_{2}$. A subset $w=\left\{x_{i_{1}}, \ldots, x_{i_{r}}\right\}$ of $V(G)$ is said to be a vertex cover of $G$ if $w \cap e \neq \varnothing$ for every $e \in E(G)$. A vertex cover is said to be minimal if it is minimal with respect to inclusion.

Let $G$ be a graph with $V(G)=\left\{x_{1}, \ldots, x_{n}\right\}$. Let $K$ be a field and $R=K\left[x_{1}, \ldots, x_{n}\right]$. The edge ideal of $G$ is defined to be $I(G)=\left\langle\left\{x_{i} x_{j}:\left\{x_{i}, x_{j}\right\} \in E(G)\right\}\right\rangle \subset R$ and the cover ideal of $G$ is defined to be $J_{G}=\left\langle x_{i_{1}}, \ldots, x_{i_{r}}:\left\{x_{i_{1}}, \ldots, x_{i_{r}}\right\}\right.$ is a (minimal) vertex cover of $\left.G\right\rangle$ It can also be seen that $J_{G}$ is the Alexander dual of $I(G)$.

Let $S=R / I$, where $R$ is a polynomial ring over $K$ and $I$ a homogeneous ideal of $R$. For a finitely generated graded $S$-module $M=\oplus M_{i}$, set

$$
t_{i}^{S}(M)=\max \left\{j: \operatorname{Tor}_{i}^{S}(M, K)_{j} \neq 0\right\},
$$

with $t_{i}^{S}(M)=-\infty$ if $\operatorname{Tor}_{i}^{S}(M, K)=0$. The Castelnuovo-Mumford regularity, denoted by $\operatorname{reg}_{S}(M)$, of an $S$-module $M$ is defined to be

$$
\operatorname{reg}_{S} M=\max \left\{t_{i}^{S}(M)-i: i \geq 0\right\}
$$

\section{Bipartite Graphs}

In this section, we study the regularity of powers of cover ideals of certain bipartite graphs. We begin with a simple observation concerning the vertex covers of a bipartite graph.

Proposition 1. Let $G$ be a bipartite graph on $n+m$ vertices. Then $G$ is a complete bipartite graph if and only if $J_{G}$ is generated by a regular sequence that has disjoint supports.

Proof. Let $V(G)=X \sqcup Y$ be the partition of the vertex set of $G$ with $X=\left\{x_{1}, \ldots, x_{n}\right\}$ and $Y=\left\{y_{n+1}, \ldots, y_{n+m}\right\}$. First, note that $J_{G}$ is generated by a regular sequence if and only if for any two minimal vertex covers $w, w^{\prime}, w \cap w^{\prime}=\varnothing$. If $G=K_{n, m}$, then $J_{G}=\left(x_{1} \cdots x_{n}, y_{n+1} \cdots y_{n+m}\right)$ which is a regular sequence. Conversely, suppose $G$ is not a complete bipartite graph. As $G$ is a bipartite graph, note that $\prod_{x_{i} \in X} x_{i}, \prod_{y_{j} \in Y} y_{j} \in J_{G}$ are minimal generators of $J_{G}$. Therefore, there exist $x_{i_{0}} \in X$ and $y_{i_{0}} \in Y$ such that $\left\{x_{i_{0}}, y_{i_{0}}\right\} \notin E(G)$. Then $w=\left\{x_{i}, y_{j}: i \neq i_{0}\right.$ and $\left.y_{j} \in N_{G}\left(x_{i_{0}}\right)\right\}$ is a minimal vertex cover of $G$ that intersects $X$ as well as $Y$ nontrivially. Therefore $J_{G}$ is not a complete intersection.

First we discuss the regularity of powers of cover ideals of complete bipartite graphs. As the cover ideal of a complete bipartite graph is a complete intersection, the result is a consequence of (Theorem 2.1 in the work by the authors of [3]).

Remark 1. Let $J=J_{K_{m, n}}$ be the cover ideal of the complete bipartite graph $K_{m, n}, m \leq n$. Then $\operatorname{reg}\left(J^{s}\right)=$ $s n+m-1$ for all $s \geq 1$.

Proof. Consider the ideal $I=\left(T_{1}, T_{2}\right) \subset R=K\left[T_{1}, T_{2}\right]$ with $\operatorname{deg} T_{1}=m$ and $\operatorname{deg} T_{2}=n$. It follows from (Theorem 2.1 in the work by the authors of [3]) that the resolution of $I^{s}$ is

$$
0 \rightarrow \bigoplus_{\substack{a_{1}+a_{2}=s+1 \\ a_{i} \geq 1}} R\left(-a_{1} m-a_{2} n\right) \rightarrow \bigoplus_{a_{1}+a_{2}=s} R\left(-a_{1} m-a_{2} n\right) \rightarrow I^{s} \rightarrow 0
$$


Note that $J=\left(x_{1} \cdots x_{m}, y_{m+1} \cdots y_{m+n}\right)$. Set $x_{1} \cdots x_{m}=T_{1}$ and $y_{m+1} \cdots y_{m+n}=T_{2}$. Then, $J^{s}$ has the minimal free resolution as in (1). If $m \leq n$, then $\operatorname{reg}\left(J^{s}\right)=s n+m-1$.

It follows from Remark 1 that in the case of the complete bipartite graph $K_{m, n}$, the stability index is 1 and the constant term is $\tau-1$, where $\tau$ is the size of a minimum vertex cover.

If the graph is not a complete bipartite graph, then the cover ideal is no longer a complete intersection. If $G$ is a Cohen-Macaulay bipartite graph, then it was shown by F. Mohammadi and S. Moradi that the vertex cover ideals are weakly polymatroidal. Therefore, they have linear quotients and hence all the powers have linear resolution (Theorem 2.2 in the work by the authors of [12]). It would be quite a challenging task to obtain the syzygies and Betti numbers of powers of cover ideals of all bipartite graphs. Therefore, we restrict our attention to some structured subclasses of bipartite graphs.

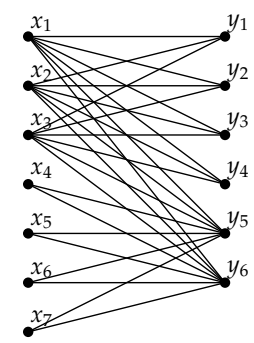

Figure 1. $K_{U_{1}, V} \cup K_{U_{2}, V_{2}}$.

Notation 1. For disjoint vertex sets $U$ and $V$, let $K_{U, V}$ denote the complete bipartite graph on $U \sqcup V$. Let $U_{1}=\left\{x_{1}, \ldots, x_{k}\right\}, U_{2}=\left\{x_{k+1}, \ldots, x_{n}\right\}, V_{1}=\left\{y_{1}, \ldots, y_{r}\right\}$, and $V_{2}=\left\{y_{r+1}, \ldots, y_{m}\right\}$. Let $U=$ $U_{1} \cup U_{2}$ and $V=V_{1} \cup V_{2}$. In the following, we consider the bipartite graph $G$ on the vertex set $U \sqcup V$ with edges $E(G)=E\left(K_{U_{1}, V}\right) \cup E\left(K_{U_{2}, V_{2}}\right)$. An illustrative figure can be seen in Figure 1 above. Note that although the vertex sets of $K_{U_{1}, V}$ and $K_{U_{2}, V_{2}}$ are not disjoint, the edge sets are. The figure on the left is an example of such a graph with $U_{1}=\left\{x_{1}, x_{2}, x_{3}\right\}, U_{2}=\left\{x_{4}, x_{5}, x_{6}, x_{7}\right\}, V_{1}=\left\{y_{1}, y_{2}, y_{3}, y_{4}\right\}$ and $V_{2}=\left\{y_{5}, y_{6}\right\}$.

Theorem 1. Let $U=U_{1} \sqcup U_{2}$ and $V=V_{1} \sqcup V_{2}$ be a collection of vertices with $|U|=n$, $\left|U_{i}\right|=n_{i},|V|=m,\left|V_{i}\right|=m_{i}$ and $1 \leq n_{i}, m_{i}$ for $i=1,2$. Let $G$ be the bipartite graph $K_{U_{1}, V} \cup K_{U_{2}, V_{2}}$. Let $R=K\left[x_{1}, \ldots, x_{n}, y_{1}, \ldots, y_{m}\right]$. Let $J_{G} \subset R$ denote the cover ideal of $G$. Then the graded minimal free resolution of $R / J_{G}$ is of the form

$$
0 \longrightarrow R\left(-n-m_{2}\right) \oplus R\left(-m-n_{1}\right) \longrightarrow R\left(-\left(n_{1}+m_{2}\right)\right) \oplus R(-m) \oplus R(-n) \longrightarrow R .
$$

In particular,

$$
\operatorname{reg}\left(J_{G}\right)=\max \left\{n+m_{2}-1, m+n_{1}-1\right\}
$$

Proof. It can easily be seen that the cover ideal $J_{G}$ is generated by $g_{1}=x_{1} \cdots x_{n}, g_{2}=y_{1} \cdots y_{m}$, and $g_{3}=x_{1} \cdots x_{n_{1}} y_{m_{1}+1} \cdots y_{m}$. Set $X_{1}=x_{1} \cdots x_{n_{1}} ; X_{2}=x_{n_{1}+1} \cdots x_{n} ; Y_{1}=y_{1} \cdots y_{m_{1}}$ and $Y_{2}=y_{m_{1}+1} \cdots y_{m}$. Then we can write $g_{1}=X_{1} X_{2}, g_{2}=Y_{1} Y_{2}$ and $g_{3}=X_{1} Y_{2}$.

Consider the minimal graded free resolution of $R / J_{G}$ over $R$ :

$$
\cdots \longrightarrow F \stackrel{\partial_{2}}{\longrightarrow} R(-n) \oplus R(-m) \oplus R\left(-\left(n_{1}+m_{2}\right)\right) \stackrel{\partial_{1}}{\longrightarrow} R,
$$

where $\partial_{1}\left(e_{1}\right)=g_{1}, \partial_{1}\left(e_{2}\right)=g_{2}$, and $\partial_{1}\left(e_{3}\right)=g_{3}$.

Let $a e_{1}+b e_{2}+c e_{3} \in \operatorname{ker} \partial_{1}$. Then $a X_{1} X_{2}+b Y_{1} Y_{2}+c X_{1} Y_{2}=0$. Solving the above equation, it can be seen that,

$$
\operatorname{ker} \partial_{1}=\operatorname{Span}_{R}\left\{Y_{2} e_{1}-X_{2} e_{3}, X_{1} e_{2}-Y_{1} e_{3}\right\}
$$


Also, it is easily verified that these two generators are $R$-linearly independent. Therefore, ker $\partial_{1} \cong$ $R^{2}$. Note that $\operatorname{deg}\left(Y_{2} e_{1}-X_{2} e_{3}\right)=n+m_{2}$ and $\operatorname{deg}\left(X_{1} e_{2}-Y_{1} e_{3}\right)=n_{1}+m$. Therefore, we get the minimal free resolution of $R / J_{G}$ as

$$
0 \longrightarrow R\left(-n-m_{2}\right) \oplus R\left(-m-n_{1}\right) \stackrel{\partial_{2}}{\longrightarrow} R\left(-\left(n_{1}+m_{2}\right)\right) \oplus R(-m) \oplus R(-n) \stackrel{\partial_{1}}{\longrightarrow} R,
$$

where $\partial_{2}(a, b)=a\left(Y_{2} e_{1}-X_{2} e_{3}\right)+b\left(X_{1} e_{2}-Y_{1} e_{3}\right)$. The regularity assertion follows immediately from the resolution.

Our aim is to compute the syzygies and the Betti numbers of $J_{G}^{s}$, where $J_{G}$ is the cover ideal discussed in Theorem 1. For this purpose, we first study the resolution of powers of the ideal $\left(X_{1} X_{2}, X_{1} Y_{2}, Y_{1} Y_{2}\right)$ and obtain the resolution and regularity of the cover ideal as a consequence. It is known from the work by the authors of [5] that all the powers of this ideal has a linear resolution. We explicitly compute the syzygies and Betti numbers for the powers of this ideal.

Theorem 2. Let $R=K\left[X_{1}, X_{2}, Y_{1}, Y_{2}\right]$ and $J=\left(X_{1} X_{2}, X_{1} Y_{2}, Y_{1} Y_{2}\right)$ be an ideal of $R$. Then, for $s \geq 2$, the minimal free resolution of $R / J^{s}$ is of the form

$$
0 \longrightarrow R^{\left(\begin{array}{c}
s \\
2
\end{array}\right)} \longrightarrow R^{2\left(\begin{array}{c}
s+1 \\
2
\end{array}\right)} \longrightarrow R^{\left(\begin{array}{c}
s+2 \\
2
\end{array}\right)} \longrightarrow R,
$$

and $\operatorname{reg}\left(J^{s}\right)=2 s$.

Proof. Denote the generators of $J$ by $g_{1}=X_{1} X_{2}, g_{2}=X_{1} Y_{2}$, and $g_{3}=Y_{1} Y_{2}$. Note that $J$ is the edge ideal of $P_{4}$, the path graph on the vertices $\left\{X_{1}, X_{2}, Y_{1}, Y_{2}\right\}$. Since $P_{4}$ is chordal, it follows that $R / J^{s}$ has a linear resolution for all $s \geq 1$ [5]. Now we compute the Betti number of the $R / J^{s}$. Write

$$
\left(g_{1}, g_{2}, g_{3}\right)^{s}=\left(g_{1}^{s}, g_{1}^{s-1}\left(g_{2}, g_{3}\right), g_{1}^{s-2}\left(g_{2}, g_{3}\right)^{2}, \ldots, g_{1}\left(g_{2}, g_{3}\right)^{s-1},\left(g_{2}, g_{3}\right)^{s}\right)
$$

where

$$
\left(g_{2}, g_{3}\right)^{t}=\left(g_{2}^{t}, g_{2}^{t-1} g_{3}, g_{2}^{t-2} g_{3}^{2}, \ldots, g_{2} g_{3}^{t-1}, g_{3}^{t}\right) .
$$

For $i \geq j$, set $M_{i, j}=g_{1}^{s-i} g_{2}^{i-j} g_{3}^{j}=\left(X_{1} X_{2}\right)^{s-i} Y_{2}^{i} Y_{1}^{j} X_{1}^{i-j}$. It follows that $\mu\left(J^{s}\right)=\frac{(s+1)(s+2)}{2}$.

Set $\beta_{1}=\frac{(s+1)(s+2)}{2}$. Let $\left\{e_{p, q} \mid 0 \leq p \leq s ; 0 \leq q \leq p\right\}$ denote the standard basis for $R^{\beta_{1}}$. Let $\partial_{1}: R^{\beta_{1}} \longrightarrow R$ be the map $\partial_{1}\left(e_{p, q}\right)=M_{p, q}$. As $g_{i}$ 's are monomials, the kernel is generated by binomials of the form $m_{i, j} M_{i, j}-m_{k, l} M_{k, l}$, where $m_{p, q}$ 's are monomials in $R$. Since the resolution of $R / J^{S}$ is linear, it is enough to find the linear syzygy relations among the generators of $\operatorname{ker}\left(\partial_{1}\right)$. To find these linear syzygies, we need to find conditions on $i, j, k, l$ such that $\frac{M_{i, j}}{M_{k, l}}$ is equal to $\frac{X_{p}}{Y_{q}}$ or $\frac{Y_{q}}{X_{p}}$ for some $p, q$. First of all, note that for such linear syzygies, $|i-k|,|j-l| \leq 1$. If $i=k$ and $j=l+1$, then $\frac{M_{i, j}}{M_{i, j+1}}=\frac{g_{3}}{g_{2}}=\frac{Y_{1}}{X_{1}}$. We get the same relation if $i=k$ and $j=l-1$. If $i=k+1$ and $j=l$, then $\frac{M_{i, j}}{M_{i-1, j}}=\frac{g_{2}}{g_{1}}=\frac{Y_{2}}{X_{2}}$. As before, $i=k-1$ yields the same relation. Therefore, the kernel is minimally generated by

$$
\left\{Y_{1} e_{i, j}-X_{1} e_{i, j+1}, X_{2} e_{i, j}-Y_{2} e_{i-1, j} \mid 0 \leq j<i \leq s\right\}
$$

Hence, $\mu\left(\operatorname{ker} \partial_{1}\right)=2\left(\begin{array}{c}s+1 \\ 2\end{array}\right)$. Write the basis elements of $R^{2\left(\begin{array}{c}s+1 \\ 2\end{array}\right)}$ as

$$
\left\{e_{1, i, p}, e_{2, i, q} \mid 1 \leq i \leq s, 0 \leq p<i \text { and } 0 \leq q<i\right\}
$$


and define $\partial_{2}: R^{2\left(\begin{array}{c}s+1 \\ 2\end{array}\right)} \longrightarrow R^{\beta_{1}}$ by

$$
\begin{aligned}
& \partial_{2}\left(e_{1, i, p}\right)=Y_{1} e_{i, p}-X_{1} e_{i, p+1} \\
& \partial_{2}\left(e_{2, i, q}\right)=X_{2} e_{i, q}-Y_{2} e_{i-1, q} .
\end{aligned}
$$

By (Proposition 3.2 in the work by the authors of [13]), $\operatorname{pdim}\left(R / J^{s}\right)=3$ for all $s \geq 2$. Hence we conclude that the minimal graded free resolution of $R / J^{s}$ is of the form

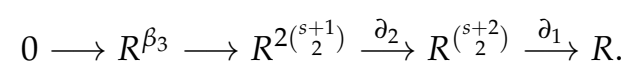

Therefore,

$$
\beta_{3}-2\left(\begin{array}{c}
s+1 \\
2
\end{array}\right)+\left(\begin{array}{c}
s+2 \\
2
\end{array}\right)-1=0
$$

so that $\beta_{3}=\left(\begin{array}{l}s \\ 2\end{array}\right)$. Now we compute the generators of the second syzygy. Again, as the resolution is linear, it is enough to compute linear generators. First, note that

$$
B=\left\{Y_{2} e_{1, i, j}-X_{2} e_{1, i+1, j}+Y_{1} e_{2, i+1, j}-X_{1} e_{2, i+1, j+1} \mid 0 \leq j<i<s\right\} \subseteq \operatorname{ker} \partial_{2} .
$$

It can easily be verified that $B$ is $R$-linearly independent. As $\mu\left(\operatorname{ker} \partial_{2}\right)=\left(\begin{array}{l}s \\ 2\end{array}\right), B$ generates ker $\partial_{2}$.

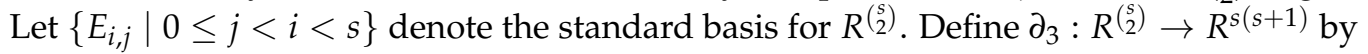

$$
\partial_{3}\left(E_{i, j}\right)=Y_{2} e_{1, i, j}-X_{2} e_{1, i+1, j}+X_{1} e_{2, i+1, j}-Y_{1} e_{2, i+1, j+1} .
$$

Therefore, we get the minimal free resolution of $R / J^{s}$ as

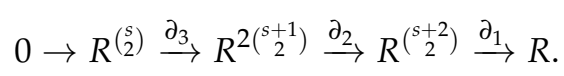

As the resolution is linear, $\operatorname{reg} J^{s}=2 s$.

As an immediate application of the previous theorem, we obtain resolution and regularity of powers of the cover ideals of graphs discussed in Theorem 1.

Corollary 1. Let $U=U_{1} \sqcup U_{2}$ and $V=V_{1} \sqcup V_{2}$ be a collection of vertices with $|U|=n,\left|U_{i}\right|=n_{i}$, $|V|=m,\left|V_{i}\right|=m_{i}$ and $1 \leq n_{i}, m_{i}$ for $i=1,2$. Let $G$ be the bipartite graph $K_{U_{1}, V} \cup K_{U_{2}, V_{2}}$. Let $R=K\left[x_{1}, \ldots, x_{n}, y_{1}, \ldots, y_{m}\right]$. Let $J_{G} \subset R$ denote the cover ideal of $G$. Then the minimal free resolution of $R / J_{G}^{s}$ is of the form

$$
0 \longrightarrow R^{\left(\begin{array}{c}
s \\
2
\end{array}\right)} \longrightarrow R^{2\left(\begin{array}{c}
(s+1 \\
2
\end{array}\right)} \longrightarrow R^{\left(\begin{array}{c}
(s+2 \\
2
\end{array}\right)} \longrightarrow R .
$$

Moreover,

$$
\operatorname{reg} J_{G}^{s}=\max \left\{\begin{array}{ll}
(s-j) n_{1}+(s-i) n_{2}+j m_{1}+i m_{2} & \text { for } 0 \leq j \leq i \leq s \\
(s-j) n_{1}+(s-i) n_{2}+(j+1) m_{1}+i m_{2}-1 & \text { for } 0 \leq j<i \leq s \\
(s-j) n_{1}+(s-i+1) n_{2}+j m_{1}+i m_{2}-1 & \text { for } 0 \leq j<i \leq s \\
(s-j) n_{1}+(s-i) n_{2}+(j+1) m_{1}+(i+1) m_{2}-2 & \text { for } 0 \leq j<i<s
\end{array} .\right.
$$

Proof. Let $R=K\left[x_{1}, \ldots, x_{n}, y_{1}, \ldots, y_{m}\right]$. Then $J_{G}=\left(x_{1} \cdots x_{n}, y_{1} \cdots y_{m}, x_{1} \cdots x_{n_{1}} y_{m_{1}+1} \cdots y_{m}\right)$. Set $X_{1}=x_{1} \cdots x_{n_{1}}, X_{2}=x_{n_{1}+1} \cdots x_{n}, Y_{1}=y_{1} \cdots y_{m_{1}}$ and $Y_{2}=y_{m_{1}+1} \cdots y_{m}$. Then $J_{G}=$ $\left(X_{1} X_{2}, Y_{1} Y_{2}, X_{1} Y_{2}\right)$. Therefore, it follows from Theorem 1 that $J_{G}^{s}$ has the given minimal free resolution. 
To compute the regularity, we need to obtain the degrees of the syzygies. Following the notation in the proof of Theorem 1, we can see that

$$
\begin{aligned}
\operatorname{deg} e_{i, j} & =(s-j) n_{1}+(s-i) n_{2}+j m_{1}+i m_{2}, \\
\operatorname{deg} e_{1, i, j} & =(s-j) n_{1}+(s-i) n_{2}+(j+1) m_{1}+i m_{2}, \\
\operatorname{deg} e_{2, i, j} & =(s-j) n_{1}+(s-i+1) n_{2}+j m_{1}+i m_{2}, \\
\operatorname{deg} E_{i, j} & =(s-j) n_{1}+(s-i) n_{2}+(j+1) m_{1}+(i+1) m_{2} .
\end{aligned}
$$

Therefore, the assertion on the regularity follows.

\subsection{Discussion}

It has been proved by Hang and Trung [11] that if $G$ is a bipartite graph on $n$ vertices and $J_{G}$ is the cover ideal of $G$, then there exists a non-negative integer $e$, such that for $s \geq n+2, \operatorname{reg}\left(J_{G}^{s}\right)=$ $\operatorname{deg}\left(J_{G}\right) s+e$, where $\operatorname{deg}\left(J_{G}\right)$ denote the maximal degree of minimal monomial generators of $J_{G}$. It follows from Remark 1 that if $G=K_{n, m}$ with $n \geq m$, then $e=m-1$ and the index of stability is 1 . If the graph is not a complete bipartite graph, then $e$ does not uniformly represent a combinatorial invariant associated to the graph as can be seen in the computations below. We compute the polynomial $\operatorname{reg}\left(J_{G}^{s}\right)$ for some classes of bipartite graphs that are considered in Corollary 1 . We see that $e$ depends on the relation between the integers $n_{1}, n_{2}, m_{1}$ and $m_{2}$. Just to illustrate the computation of the polynomial from Corollary 1 , we compute $\operatorname{reg}\left(J_{G}^{s}\right)$ in some cases below:

1. If $m_{1}=m_{2}=1$, then it can be seen that for $s \geq 2$,

$$
\operatorname{reg}\left(J_{G}^{s}\right)=\max \left\{(s-j) n_{1}+(s-i+1) n_{2}+j m_{1}+i m_{2}-1: 0 \leq j<i \leq s\right\} .
$$

Since $(s-j) n_{1}+(s-i+1) n_{2}+j m_{1}+i m_{2}-1=s\left(n_{1}+n_{2}\right)+j\left(1-n_{1}\right)+i\left(1-n_{2}\right)+n_{2}-1$ and $n_{1}>1, n_{2}>1$, this expression attains maximum when $i$ and $j$ attain minimum, i.e., if $j=0$ and $i=1$. Therefore, $\operatorname{reg}\left(J_{G}^{s}\right)=n s$. Thus, in this case, $e=0$. It can also be noted that, since $n \geq 2$, it follows from Theorem 1 that $\operatorname{reg}\left(J_{G}\right)=n$. Therefore, in this case, the stability index is also equal to 1.

2. If $n_{1}=n_{2}=m_{1}=m_{2}=\ell>1$, then for $s \geq 2$,

$$
\operatorname{reg}\left(J_{G}^{s}\right)=\max \left\{(s-j) n_{1}+(s-i) n_{2}+(j+1) m_{1}+(i+1) m_{2}-2: 0 \leq j<i \leq s\right\} .
$$

Therefore, $\operatorname{reg}\left(J_{G}^{s}\right)=2 \ell s+(2 \ell-2)$, and therefore $e=2 \ell-2$. Note that in this case, the stability index is 2 .

3. $n_{1} \geq m_{2} \geq n_{2}=m_{1}$ : Note that, in this case, $\operatorname{deg}\left(J_{G}\right)=n_{1}+m_{2}$. We have

$$
\operatorname{reg}\left(J_{G}^{s}\right)=\max \left\{(s-j) n_{1}+(s-i) n_{2}+(j+1) m_{1}+(i+1) m_{2}-2: 0 \leq j<i<s\right\} .
$$

Since $(s-j) n_{1}+(s-i) n_{2}+(j+1) m_{1}+(i+1) m_{2}-2=s\left(n_{1}+n_{2}\right)+j\left(m_{1}-n_{1}\right)+i\left(m_{2}-n_{2}\right)+$ $m_{1}+m_{2}-2$. Since $n_{1} \geq m_{1}$ and $m_{2} \geq n_{2}$, the above expression attains the maximum when $i$ attains the maximum and $j$ attains the minimum, i.e., if $i=s-1$ and $j=0$. Therefore, $\operatorname{reg}\left(J_{G}^{s}\right)=\left(n_{1}+m_{2}\right) s+\left(n_{2}+m_{1}-2\right)$ and hence $e=n_{2}+m_{1}-2$.

4. $n_{1} \geq n_{2}=m_{1} \geq m_{2}$ : In this case, $\operatorname{deg}\left(J_{G}\right)=n$ and

$$
\operatorname{reg}\left(J_{G}^{s}\right)=\max \left\{(s-j) n_{1}+(s-i) n_{2}+(j+1) m_{1}+(i+1) m_{2}-2: 0 \leq j<i<s\right\} .
$$

As in the previous case, one can conclude that the maximum is attained when $i=1$ and $j=0$. Therefore, $\operatorname{reg}\left(J_{G}^{s}\right)=n s+\left(2 m_{2}-2\right)$. Thus $e=2 m_{2}-2$.

5. $n_{2}=m_{1} \geq n_{1} \geq m_{2}$ : As done earlier, one can conclude that reg $\left(J_{G}\right)^{s}=n s+\left(2 m_{2}-2\right)$, and therefore $e=2 m_{2}-2$. 
Remark 2. If $G=K_{U_{1}, V} \cup K_{U_{2}, V_{2}} \cup K_{U_{3}, V_{3}}$, for some set of vertices $U_{i}, V_{i}$, then one can still describe the complete resolution and the regularity of $J_{G}^{s}$ using a similar approach. However, the resulting syzygies are not so easy to describe though the generating sets are similar. Therefore, we restrict ourselves to the above discussion.

\section{Complete Multipartite Graphs}

In this section our goal is to understand the resolution and regularity of the powers of cover ideals of complete $m$-partite graphs. Let $G$ be a complete $m$-partite graph and let $J_{G}$ be the cover ideal of $G$. Let $R=K\left[x_{1}, \ldots, x_{m}\right]$. It is known that the cover ideal $J_{G}$ of complete graph $G=K_{m}$ is generated by all squarefree monomials $x_{1} x_{2} \cdots \hat{x}_{i} \cdots x_{m}$ of degree $m-1$. Moreover one can also identify this cover ideal with the squarefree Veronese ideal $I=I_{m, m-1}$, and thus it is a polymatroidal ideal [14]. Therefore, by the results of Conca-Herzog [6] and Herzog-Hibi [15], we have

Remark 3. The cover ideal $J_{G}$ of the complete graph $G=K_{m}$ has linear quotients and hence has linear resolution. Moreover $J_{G}^{s}$ has linear resolution for all $s \geq 1$.

If $I$ is an ideal of $R$ all of whose powers have linear resolution, then depth $R / I^{k}$ is a non-increasing function of $k$ and depth $R / I^{k}$ is constant for all $k \gg 0$, ([14] Proposition 2.1). Further, we have,

Remark 4. (Corollary 3.4 in the work of [14]) Let $R=K\left[x_{1}, \cdots, x_{m}\right]$ and $J_{G}$ be the cover ideal of $G=K_{m}$. Then

$$
\operatorname{depth} R / J_{G}^{s}=\max \{0, m-s-1\} \text {. }
$$

In particular, depth $R / J_{G}^{s}=0$ for all $s \geq m-1$.

\subsection{Complete Tripartite:}

We first describe the graded minimal free resolution of $J_{K_{3}}^{s}$ for all $s \geq 1$. We also obtain the Hilbert series of the powers.

Theorem 3. Let $R=K\left[x_{1}, x_{2}, x_{3}\right]$ and $I=\left(x_{1} x_{2}, x_{1} x_{3}, x_{2} x_{3}\right)$. Then, the graded minimal free resolution of $R / I$ is of the form

$$
0 \rightarrow R(-3)^{2} \rightarrow R(-2)^{3} \rightarrow R .
$$

For $s \geq 2$, the graded minimal free resolution of $R / I^{s}$ is of the form:

$$
0 \rightarrow R(-2 s-2)^{\left(\begin{array}{c}
s \\
2
\end{array}\right)} \rightarrow R(-2 s-1)^{2\left(\begin{array}{c}
s+1 \\
2
\end{array}\right)} \rightarrow R(-2 s)^{\left(\begin{array}{c}
s+2 \\
2
\end{array}\right)} \rightarrow R
$$

so that $\operatorname{reg}_{R}\left(I^{s}\right)=2$ s. Moreover, the Hilbert series of $R / I^{s}$ is given by

$$
H\left(R / I^{s}, t\right)=\frac{1+2 t+3 t^{2}+\cdots+2 s t^{2 s-1}-\left(\left(\begin{array}{c}
s+2 \\
2
\end{array}\right)-2 s-1\right) t^{2 s}}{(1-t)} .
$$

Proof. It is clear that the resolution of $I$ is as given in the assertion of the theorem. Since $I$ is the cover ideal of $K_{3}, I^{s}$ has linear minimal free resolution for all $s \geq 1$. Note also that by ([16] Lemma 3.1), $\operatorname{depth} R / I^{s}=0$ for all $s \geq 2$ so that $\operatorname{pdim} R / I^{s}=3$ for all $s \geq 2$. Therefore, the minimal free resolution of $R / I^{S}$ is of the form

$$
0 \rightarrow R(-2 s-2)^{\beta_{3}} \stackrel{\partial_{3}}{\longrightarrow} R(-2 s-1)^{\beta_{2}} \stackrel{\partial_{2}}{\longrightarrow} R(-2 s)^{\beta_{1}} \stackrel{\partial_{1}}{\longrightarrow} R .
$$

Now we describe the syzygies and Betti numbers of $R / I^{s}$ for $s \geq 2$. Let $g_{1}=x_{1} x_{2}, g_{2}=x_{1} x_{3}$ and $g_{3}=x_{2} x_{3}$. The generators of $I^{s}$ are of the form $g_{1}^{\ell_{1}} g_{2}^{\ell_{2}} g_{3}^{\ell_{3}}$, where $0 \leq \ell_{1}, \ell_{2}, \ell_{3} \leq s$ and $\ell_{1}+\ell_{2}+\ell_{3}=s$. Set $f_{\ell_{1}, \ell_{2}, \ell_{3}}=g_{1}^{\ell_{1}} g_{2}^{\ell_{2}} g_{3}^{\ell_{3}}=x_{1}^{s-\ell_{3}} x_{2}^{s-\ell_{2}} x_{3}^{s-\ell_{1}}$. It is easy to see that $\mu\left(I^{s}\right)=\left(\begin{array}{c}s+2 \\ 2\end{array}\right)$, as the cardinality 
of a minimal generating set of $I^{s}$ is same as the total number of non-negative integral solution of $\ell_{1}+\ell_{2}+\ell_{3}=s$, which is $\left(\begin{array}{c}s+2 \\ 2\end{array}\right)$.

Let $\left\{e_{\ell_{1}, \ell_{2}, \ell_{3}}: 0 \leq \ell_{1}, \ell_{2}, \ell_{3} \leq s ; \ell_{1}+\ell_{2}+\ell_{3}=s\right\}$ denote the standard basis for $R^{\left(\begin{array}{c}s+2 \\ 2\end{array}\right)}$ and consider the map $\partial_{1}: R^{(s+2)} \rightarrow R$ defined by $\partial_{1}\left(e_{\ell_{1}, \ell_{2}, \ell_{3}}\right)=f_{\ell_{1}, \ell_{2}, \ell_{3}}$. As $f_{\ell_{1}, \ell_{2}, \ell_{3}}$ 's are monomials, the kernel is generated by binomials of the form $m_{\ell_{1}, \ell_{2}, \ell_{3}} f_{\ell_{1}, \ell_{2}, \ell_{3}}-m_{t_{1}, t_{2}, t_{3}} f_{t_{1}, t_{2}, t_{3}}$, where $m_{i, j, k}$ 's are monomials in $R$. Also, as the minimal free resolution is linear, the kernel is generated in degree 1 . Note that $\frac{f_{\ell_{1}, \ell_{2}, \ell_{3}}}{f_{t_{1}, t_{2}, t_{3}}}=x_{1}^{t_{3}-\ell_{3}} x_{2}^{t_{2}-\ell_{2}} x_{3}^{t_{1}-\ell_{1}}$. Therefore, for $\frac{f_{\ell_{1}, \ell_{2}, \ell_{3}}}{f_{t_{1}, t_{2}, t_{3}}}$ to be a linear fraction, $\left|t_{i}-\ell_{i}\right| \leq 1$ for $i=1,2,3$.

Let $t_{3}=\ell_{3}, t_{2}=\ell_{2}+1$ and $t_{1}=\ell_{1}-1$. The corresponding linear syzygy relation is

$$
x_{3} \cdot f_{\ell_{1}, \ell_{2}, \ell_{3}}-x_{2} \cdot f_{\ell_{1}-1, \ell_{2}+1, \ell_{3}}=0,
$$

where $1 \leq \ell_{1} \leq s$, and $0 \leq \ell_{2}, \ell_{3} \leq s-1$. Note that the number of such relations is equal to the number of integral solution to $\left(\ell_{1}-1\right)+\ell_{2}+\ell_{3}=s$, i.e., $\left(\begin{array}{c}s+1 \\ 2\end{array}\right)$. Similarly, if $t_{3}=\ell_{3}+1, t_{2}=\ell_{2}-1$ and $t_{1}=\ell_{1}$, then we get the corresponding linear syzygy relation as

$$
x_{2} \cdot f_{\ell_{1}, \ell_{2}, \ell_{3}}-x_{1} \cdot f_{\ell_{1}, \ell_{2}-1, \ell_{3}+1}=0,
$$

where $0 \leq \ell_{1}, \ell_{3} \leq s-1$ and $1 \leq \ell_{2} \leq s$. Note that the linear syzygy relation obtained by fixing $\ell_{2}$ and taking $\left|\ell_{i}-t_{i}\right|=1$ for $i=1,2$

$$
x_{3} \cdot f_{\ell_{1}, \ell_{2}, \ell_{3}}-x_{1} \cdot f_{\ell_{1}-1, \ell_{2}, \ell_{3}+1}=0
$$

can be obtained from Equations (2) and (3) by setting the $\ell_{i}$ 's appropriately. Therefore,

$\operatorname{ker} \partial_{1}=\left\langle x_{3} \cdot e_{\ell_{1}, \ell_{2}, \ell_{3}}-x_{2} \cdot e_{\ell_{1}-1, \ell_{2}+1, \ell_{3}}, x_{2} \cdot e_{\ell_{1}-1, \ell_{2}+1, \ell_{3}}-x_{1} \cdot e_{\ell_{1}-1, \ell_{2}, \ell_{3}+1}: 1 \leq \ell_{1} \leq s, 0 \leq \ell_{2}, \ell_{3} \leq s-1\right\rangle$.

As there are $\left(\begin{array}{c}s+1 \\ 2\end{array}\right)$ minimal generators of type (2) and (3), $\mu\left(\operatorname{ker} \partial_{1}\right)=\beta_{2}=2\left(\begin{array}{c}s+1 \\ 2\end{array}\right)$. Write the standard basis of $R^{2\left(\begin{array}{c}s+1 \\ 2\end{array}\right)}$ as

$$
B_{2}=\left\{\begin{array}{l}
e_{1,\left(\ell_{2}+1, \ell_{3}\right), \ell_{1}-1} \\
e_{2,\left(\ell_{1}, \ell_{2}\right), \ell_{3}}
\end{array}: 1 \leq \ell_{1} \leq s, 0 \leq \ell_{2}, \ell_{3} \leq s-1\right\}
$$

and define $\partial_{2}: R^{2\left(\begin{array}{c}s+1 \\ 2\end{array}\right)} \rightarrow R^{\left(\begin{array}{c}(s+2 \\ 2\end{array}\right)}$ by

$$
\begin{aligned}
\partial_{2}\left(e_{1,\left(\ell_{2}+1, \ell_{3}\right), \ell_{1}-1}\right) & =x_{2} \cdot e_{\ell_{1}-1, \ell_{2}+1, \ell_{3}}-x_{1} \cdot e_{\ell_{1}-1, \ell_{2}, \ell_{3}+1}, \\
\partial_{2}\left(e_{2,\left(\ell_{1}, \ell_{2}\right), \ell_{3}}\right) & =x_{3} \cdot e_{\ell_{1}, \ell_{2}, \ell_{3}}-x_{2} \cdot e_{\ell_{1}-1, \ell_{2}+1, \ell_{3}} .
\end{aligned}
$$

From the equation, $\beta_{3}-2\left(\begin{array}{c}s+1) \\ 2\end{array}\right)+\left(\begin{array}{c}s+2 \\ 2\end{array}\right)-1=0$, it follows that $\beta_{3}=\left(\begin{array}{l}s \\ 2\end{array}\right)$. Now, we describe ker $\partial_{2}$. For $2 \leq \ell_{1} \leq s$ and $0 \leq \ell_{2}, \ell_{3} \leq s-2$, set

$$
E_{\ell_{1}, \ell_{2}, \ell_{3}}=-x_{3} \cdot e_{2,\left(\ell_{1}-1, \ell_{2}\right), \ell_{3}+1}+x_{2} \cdot e_{2,\left(\ell_{1}-1, \ell_{2}+1\right), \ell_{3}}+x_{2} \cdot e_{1,\left(\ell_{2}+2, \ell_{3}\right), \ell_{1}-2}-x_{3} \cdot e_{1,\left(\ell_{2}+1, \ell_{3}\right), \ell_{1}-1} \text {. }
$$

Note that

$$
B_{3}=\left\{E_{\ell_{1}, \ell_{2}, \ell_{3}}: 2 \leq \ell_{1} \leq s, 0 \leq \ell_{2}, \ell_{3} \leq s-2\right\} \subset \operatorname{ker} \partial_{2} .
$$

Let $N=\operatorname{ker} \partial_{2}$. We claim that $\bar{B}_{3}=\left\{\bar{E}_{\ell_{1}, \ell_{2}, \ell_{3}}\right\}$ is a basis for $N / \mathfrak{m} N$. It is enough to prove that $\bar{B}_{3}$ is $R / \mathfrak{m}$-linearly independent. Suppose $\sum_{\ell_{1}, \ell_{2}, \ell_{3}} \alpha_{\ell_{1}, \ell_{2}, \ell_{3}} \bar{E}_{\ell_{1}, \ell_{2}, \ell_{3}}=\overline{0}$. Since $B_{3} \subset N_{1}$ and $\mathfrak{m} N \subset \oplus_{r \geq 2} N_{r}$, the above equation implies that $\sum \alpha_{\ell_{1}, \ell_{2}, \ell_{3}} E_{\ell_{1}, \ell_{2}, \ell_{3}}=0$. In the above equation, the coefficient of $e_{2,(i, j), k}=-x_{3} \alpha_{i+1, j, i-1}+x_{2} \alpha_{i+1, j-1, k}$ and coefficient of $e_{1,(i, j), k}=x_{2} \alpha_{k+2, j-2, i}-x_{3} \alpha_{k+1, j-1, i}$. As the set $B_{2}$ is linearly independent in $R^{2\left(\begin{array}{c}(+1 \\ 2\end{array}\right)}$, all of these coefficients have to be zero, i.e., $\alpha_{i, j, k}=0$ for all $i, j, k$. 
This proves our claim and hence $N=\left\langle B_{3}\right\rangle$. Let $\left\{H_{\ell_{1}, \ell_{2}, \ell_{3}}: 2 \leq \ell_{1} \leq s, 0 \leq \ell_{2}, \ell_{3} \leq s-2\right\}$ denote the standard basis of $R^{\left(\frac{s}{2}\right)}$. Define $\partial_{3}: R^{\left(\frac{s}{2}\right)} \rightarrow R^{2\left(\frac{(s+1)}{2}\right)}$ by $\partial_{3}\left(H_{\ell_{1}, \ell_{2}, \ell_{3}}\right)=E_{\ell_{1}, \ell_{2}, \ell_{3}}$. As $\operatorname{pdim}\left(R / I^{s}\right)=3$, this map is injective and the resolution is complete.

It can also be seen that $\operatorname{deg} e_{\ell_{1}, \ell_{2}, \ell_{3}}=2 s, \operatorname{deg} e_{1,\left(\ell_{2}+1, \ell_{3}\right), \ell_{1}-1}=\operatorname{deg} e_{2,\left(\ell_{1}, \ell_{2}\right), \ell_{3}}=2 s+1$ and $\operatorname{deg} H_{\ell_{1}, \ell_{2}, \ell_{3}}=2 s+2$. Therefore, we get the minimal graded free resolution of $R / I^{s}$ as

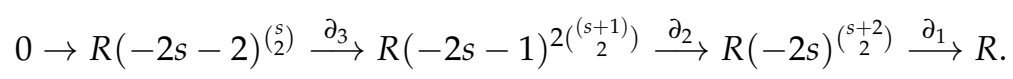

Therefore, the Hilbert series of $R / I^{s}$ is given by

$$
\begin{aligned}
H\left(R / I^{s}, t\right) & =\frac{1-\left(\begin{array}{c}
s+2 \\
2
\end{array}\right) t^{2 s}+2\left(\begin{array}{c}
s+1 \\
2
\end{array}\right) t^{2 s+1}-\left(\begin{array}{c}
s \\
2
\end{array}\right) t^{2 s+2}}{(1-t)^{3}} \\
& =(1-t)^{-2} \frac{\left(1-\left(\begin{array}{c}
s+2 \\
2
\end{array}\right) t^{2 s}+2\left(\begin{array}{c}
s+1 \\
2
\end{array}\right) t^{2 s+1}-\left(\begin{array}{c}
s \\
2
\end{array}\right) t^{2 s+2}\right)}{(1-t)} .
\end{aligned}
$$

By expanding $(1-t)^{-2}$ in the power series form and multiplying with the numerator, we get the required expression.

We now proceed to compute the minimal graded free resolution of powers of complete tripartite graphs.

Notation 2. Let $G$ denote a complete tripartite graph with $V(G)=V_{1} \sqcup V_{2} \sqcup V_{3}$ and $E(G)=\{\{a, b\}: b \in$ $\left.V_{i}, b \in V_{j}, i \neq j\right\}$. Set $V_{1}=\left\{x_{1}, \ldots, x_{\ell}\right\}, V_{2}=\left\{y_{1}, \ldots, y_{m}\right\}$ and $V_{3}=\left\{z_{1}, \ldots, z_{n}\right\}$. Let $J_{G}$ denote the vertex cover ideal of $G$. Let $X=\prod_{i=1}^{\ell} x_{i}, Y=\prod_{j=1}^{m} y_{i}$ and $Z=\prod_{k=1}^{n} z_{i}$. It can be seen that $J_{G}=(X Y, X Z, Y Z)$.

Theorem 4. Let $R=K\left[x_{1}, \ldots x_{m_{1}}, y_{1}, \ldots, y_{m_{2}}, z_{1}, \ldots, z_{m_{3}}\right]$. Let $G$ be a complete tripartite graph as in Notation 2. Let $J_{G} \subset R$ denote the cover ideal of $G$. Then for all $s \geq 2$, the minimal free resolution of $R / J_{G}^{s}$ is of the form

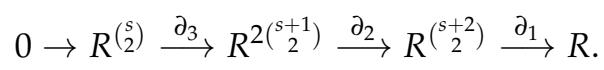

Set $\alpha=\left(s-\ell_{3}\right) m_{1}+\left(s-\ell_{2}\right) m_{2}+\left(s-\ell_{1}\right) m_{3}$. Then for all $s \geq 2$,

$$
\operatorname{reg}\left(J_{G}^{s}\right)=\max \begin{cases}\alpha, & \text { for } 0 \leq \ell_{1}, \ell_{2}, \ell_{3} \leq s, \\ \alpha+m_{3}-1, & \text { for } 1 \leq \ell_{1} \leq s, 0 \leq \ell_{2}, \ell_{3} \leq s-1, \\ \alpha+2 m_{3}-2, & \text { for } 2 \leq \ell_{1} \leq s, 0 \leq \ell_{2}, \ell_{3} \leq s-2 .\end{cases}
$$

Proof. Taking $X_{1}=x_{1}, X_{2}=x_{2}$ and $X_{3}=x_{3}$ in Theorem 3 , it follows that the minimal free resolution of $S / J_{G}^{s}$ is of the given form:

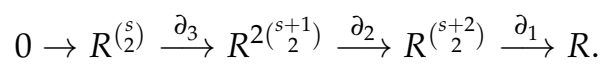

We now compute the degrees of the generators and hence obtain the regularity. Let $\operatorname{deg} X_{1}=m_{1}$, $\operatorname{deg} X_{2}=m_{2}$, and $\operatorname{deg} X_{3}=m_{3}$. Then, it follows that

$$
\operatorname{deg} e_{\ell_{1}, \ell_{2}, \ell_{3}}=\operatorname{deg}\left(X_{1}^{s-\ell_{3}} X_{2}^{s-\ell_{3}} X_{3}^{s-\ell_{1}}\right)=\left(s-\ell_{3}\right) m_{1}+\left(s-\ell_{2}\right) m_{2}+\left(s-\ell_{1}\right) m_{3} .
$$

We observe that

$$
\begin{gathered}
\operatorname{deg} e_{1,\left(\ell_{2}+1, \ell_{3}\right), \ell_{1}-1}=\operatorname{deg} e_{2,\left(\ell_{1}, \ell_{2}\right), \ell_{3}}=\operatorname{deg} e_{\ell_{1}, \ell_{2}, \ell_{3}}+\operatorname{deg} X_{3} \\
\operatorname{deg} H_{\ell_{1}, \ell_{2}, \ell_{3}}=\operatorname{deg} e_{\ell_{1}, \ell_{2}, \ell_{3}}+2 \operatorname{deg} X_{3} .
\end{gathered}
$$


Therefore,

$$
\operatorname{reg}\left(J_{G}^{s}\right)=\max \begin{cases}\operatorname{deg} e_{\ell_{1}, \ell_{2}, \ell_{3}}, & \text { for } 0 \leq \ell_{1}, \ell_{2}, \ell_{3} \leq s, \\ \operatorname{deg} e_{\ell_{1}, \ell_{2}, \ell_{3}}+\operatorname{deg}\left(X_{3}\right)-1, & \text { for } 1 \leq \ell_{1} \leq s, 0 \leq \ell_{2}, \ell_{3} \leq s-1, \\ \operatorname{deg} e_{\ell_{1}, \ell_{2}, \ell_{3}}+2 \operatorname{deg}\left(X_{3}\right)-2, & \text { for } 2 \leq \ell_{1} \leq s, 0 \leq \ell_{2}, \ell_{3} \leq s-2,\end{cases}
$$

where $\ell_{1}+\ell_{2}+\ell_{3}=s$. Let $\alpha=\operatorname{deg} e_{\ell_{1}, \ell_{2}, \ell_{3}}$. Then the regularity assertion follows.

Observe that the above expression for $\operatorname{reg}\left(J_{G}^{s}\right)$ is not really in the form $d s+e$. Given a graph, one can compute $d$ and $e$ by studying the interplay between the cardinality of the partitions. For example, suppose the graph is unmixed, i.e., all the partitions are of same cardinality. Then, $\operatorname{reg}\left(J_{G}^{s}\right)=$ $2 \ell s+(2 \ell-2)$ for all $s \geq 2$, where $\ell=m_{1}=m_{2}=m_{3}$. Note also that the stabilization index in this case is 2 . As in Section 3.1, one can derive various expressions for reg $\left(J_{G}^{s}\right)$ for different cases as well. Consider the arithmetic progression $m_{1}=m+2 r, m_{2}=m$, and $m_{3}=m+r$ :

Corollary 2. Let $m, r$ be any two positive integers. Let $m_{1}=m+2 r, m_{2}=m$, and $m_{3}=m+r$ in Theorem 4 . Then for all $s \geq 2$, we have

$$
\operatorname{reg}\left(J_{G}^{s}\right)=s(2 m+3 r)+2 m-2
$$

Proof. By Theorem $4, \alpha=\left(s-\ell_{3}\right) m_{1}+\left(s-\ell_{2}\right) m_{2}+\left(s-\ell_{1}\right) m_{3}$. Therefore, we get

$$
\alpha=s(3 m+3 r)-\ell_{3}(m+2 r)-\ell_{2}(m)-\ell_{1}(m+r) .
$$

Using Theorem 4, we have for all $s \geq 2$,

$$
\operatorname{reg}\left(J_{G}^{s}\right)=\max \begin{cases}\alpha, & \text { for } 0 \leq \ell_{1}, \ell_{2}, \ell_{3} \leq s \\ \alpha+(m+r)-1, & \text { for } 1 \leq \ell_{1} \leq s, 0 \leq \ell_{2}, \ell_{3} \leq s-1 \\ \alpha+2(m+r)-2, & \text { for } 2 \leq \ell_{1} \leq s, 0 \leq \ell_{2}, \ell_{3} \leq s-2\end{cases}
$$

where $\ell_{1}+\ell_{2}+\ell_{3}=s$. As regularity $\operatorname{reg}\left(J_{G}^{s}\right)$ is maximum of all the numbers, we need to maximize the value of $\alpha$. For this to happen, negative terms in $\alpha$ should be minimum. The coefficient of $\ell_{3}$ is largest among the negative terms in $\alpha$, so $\ell_{3}$ should be assigned the least value. After $\ell_{3}$, assign the minimum value to $\ell_{1}$, and finally take $\ell_{2}=s-\ell_{1}-\ell_{3}$. For example, to get the maximum of $\alpha$ when $2 \leq \ell_{1} \leq s, 0 \leq \ell_{2}, \ell_{3} \leq s-2$, put $\ell_{3}=0, \ell_{1}=2$, and $\ell_{2}=s-2$. We get for all $s \geq 2$

$$
\operatorname{reg}\left(J_{G}^{s}\right)=\max \begin{cases}s(2 m+3 r), & \text { for } 0 \leq \ell_{1}, \ell_{2}, \ell_{3}, \ell_{4} \leq s \\ s(2 m+3 r)+m-1, & \text { for } 1 \leq \ell_{1} \leq s, 0 \leq \ell_{2}, \ell_{3}, \ell_{4} \leq s-1 \\ s(2 m+3 r)+2 m-2, & \text { for } 2 \leq \ell_{1} \leq s, 0 \leq \ell_{2}, \ell_{3}, \ell_{4} \leq s-2\end{cases}
$$

For all $m \geq 1$, and for all $s \geq 2$, we get

$$
\operatorname{reg}\left(J_{G}^{s}\right)=s(2 m+3 r)+2 m-2
$$

\subsection{Complete 4-Partite Graphs}

We now describe the resolution and regularity of powers of cover ideals of 4-partite graphs. For this purpose, we first study the resolution of powers of cover ideal of the complete graph $K_{4}$.

Theorem 5. Let $R=K\left[x_{1}, x_{2}, x_{3}, x_{4}\right]$ and $I=\left(x_{1} x_{2} x_{3}, x_{1} x_{2} x_{4}, x_{1} x_{3} x_{4}, x_{2} x_{3} x_{4}\right)$. Then, for $s \geq 3$, the minimal graded free resolution of $R / I^{s}$ is of the form

$$
0 \rightarrow R(-3 s-3)^{\beta_{4}} \longrightarrow R(-3 s-2)^{\beta_{3}} \longrightarrow R(-3 s-1)^{\beta_{2}} \longrightarrow R(-3 s)^{\beta_{1}} \longrightarrow R,
$$


where

$$
\beta_{1}=\left(\begin{array}{c}
s+3 \\
3
\end{array}\right), \beta_{2}=3\left(\begin{array}{c}
s+2 \\
3
\end{array}\right), \beta_{3}=3\left(\begin{array}{c}
s+1 \\
3
\end{array}\right), \text { and } \beta_{4}=\left(\begin{array}{l}
s \\
3
\end{array}\right) \text {. }
$$

In particular, $\operatorname{reg}_{R}\left(I^{S}\right)=3$ s. Moreover the Hilbert series of $R / I^{s}$ is given by

$$
\begin{aligned}
H\left(R / I^{s}, t\right) & =\frac{1+2 t+3 t^{2}+4 t^{3}+\cdots+3 s t^{3 s-1}-\left(\left(\begin{array}{c}
s+3 \\
3
\end{array}\right)-3 s-1\right) t^{3 s}+\left(\begin{array}{c}
s \\
3
\end{array}\right) t^{3 s+1}}{(1-t)^{2}} \\
& =\frac{\sum_{i=0}^{3 s-1}(i+1) t^{i}-\left(\left(\begin{array}{c}
s+3 \\
3
\end{array}\right)-3 s-1\right) t^{3 s}+\left(\begin{array}{c}
s \\
3
\end{array}\right) t^{3 s+1}}{(1-t)^{2}} .
\end{aligned}
$$

Proof. By Remark $3, I^{s}$ has linear resolution for all $s \geq 1$. Moreover, by Remark 4 , depth $R / I^{s}=0$ for all $s \geq 3$, and therefore pdim $R / I^{s}=4$ for all $s \geq 3$. Therefore, the minimal graded free resolution of $R / I^{s}$, for $s \geq 3$, is of the form

$$
0 \rightarrow R(-3 s-3)^{\beta_{4}} \stackrel{\partial_{4}}{\longrightarrow} R(-3 s-2)^{\beta_{3}} \stackrel{\partial_{3}}{\longrightarrow} R(-3 s-1)^{\beta_{2}} \stackrel{\partial_{2}}{\longrightarrow} R(-3 s)^{\beta_{1}} \stackrel{\partial_{1}}{\longrightarrow} R .
$$

Let $g_{1}=x_{1} x_{2} x_{3}, g_{2}=x_{1} x_{2} x_{4}, g_{3}=x_{1} x_{3} x_{4}$ and $g_{4}=x_{2} x_{3} x_{4}$. The minimal generators of $I^{s}$ are of the form $g_{1}^{\ell_{1}} g_{2}^{\ell_{2}} g_{3}^{\ell_{3}} g_{4}^{\ell_{4}}$ where $0 \leq \ell_{i} \leq s$ for every $i$ and $\ell_{1}+\ell_{2}+\ell_{3}+\ell_{4}=s$. The number of non-negative integral solution to the linear equation $\ell_{1}+\ell_{2}+\ell_{3}+\ell_{4}=s$ is $\left(\begin{array}{c}s+3 \\ 3\end{array}\right)$. Therefore, we have $\mu\left(J^{s}\right)=\beta_{1}=\left(\begin{array}{c}s+3 \\ 3\end{array}\right)$. Note that $g_{1}^{\ell_{1}} g_{2}^{\ell_{2}} g_{3}^{\ell_{3}} g_{4}^{\ell_{4}}=x_{1}^{s-\ell_{4}} x_{2}^{s-\ell_{3}} x_{3}^{s-\ell_{2}} x_{4}^{s-\ell_{1}}$ with $0 \leq \ell_{1}, \ell_{2}, \ell_{3}, \ell_{4} \leq s$. Set $f_{\ell_{1}, \ell_{2}, \ell_{3}, \ell_{4}}=x_{1}^{s-\ell_{4}} x_{2}^{s-\ell_{3}} x_{3}^{s-\ell_{2}} x_{4}^{s-\ell_{1}}$. Let

$$
\left\{e_{\ell_{1}, \ell_{2}, \ell_{3}, \ell_{4}}: 0 \leq \ell_{1}, \ell_{2}, \ell_{3}, \ell_{4} \leq s \text { and } \ell_{1}+\ell_{2}+\ell_{3}+\ell_{4}=s\right\}
$$

denote the standard basis of $R^{\left(\begin{array}{c}s+3 \\ 3\end{array}\right)}$ and consider the map $\partial_{1}: R^{\left(\begin{array}{c}s+3 \\ 3\end{array}\right)} \rightarrow R$ defined by $\partial_{1}\left(e_{\ell_{1}, \ell_{2}, \ell_{3}, \ell_{4}}\right)=$ $f_{\ell_{1}, \ell_{2}, \ell_{3}, \ell_{4}}$. Now we find the minimal generators for ker $\partial_{1}$.

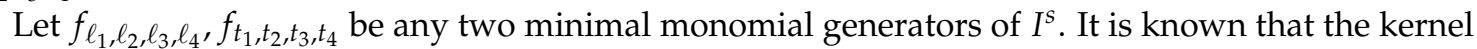
of $\partial_{1}$ is generated by binomials of the form $m_{\ell_{1}, \ell_{2}, \ell_{3}, \ell_{4}} f_{\ell_{1}, \ell_{2}, \ell_{3}, \ell_{4}}-m_{t_{1}, t_{2}, t_{3}, t_{4}} f_{t_{1}, t_{2}, t_{3}, t_{4}}$, where $m_{i, j, k, l}$ 's are monomials in $R$. As the syzygy is generated by linear binomials, we need to find conditions on $\ell_{1}, \ell_{2}, \ell_{3}, \ell_{4}$ such that $\frac{f_{\ell_{1}, \ell_{2}, \ell_{3}, \ell_{4}}}{f_{t_{1}, t_{2}, t_{3}, t_{4}}}$ is equal to $\frac{x_{i}}{x_{j}}$ for some $i, j$. Observe that

$$
\frac{f_{\ell_{1}, \ell_{2}, \ell_{3}, \ell_{4}}}{f_{t_{1}, t_{2}, t_{3}, t_{4}}}=x_{1}^{t_{4}-\ell_{4}} x_{2}^{t_{3}-\ell_{3}} x_{3}^{t_{2}-\ell_{2}} x_{4}^{t_{1}-\ell_{1}} .
$$

Suppose $t_{4}=\ell_{4}+1, t_{3}=\ell_{3}-1$ and $t_{j}=\ell_{j}$ for $j=1,2$. Then we get the linear syzygy relation

$$
x_{2} f_{\ell_{1}, \ell_{2}, \ell_{3}, \ell_{4}}-x_{1} f_{\ell_{1}, \ell_{2}, \ell_{3}-1, \ell_{4}+1}=0 \text {. }
$$

Fixing $t_{3}$ and $t_{4}$ with $1 \leq t_{3}+t_{4} \leq s$, there are as many linear syzygies are there as the number of solutions of $t_{1}+t_{2}=s-\left(t_{3}+t_{4}\right)$. Therefore, for the pair $\left(t_{3}, t_{4}\right)$, there are $\left(\begin{array}{c}s+1 \\ 2\end{array}\right)+\left(\begin{array}{c}s \\ 2\end{array}\right)+\cdots+\left(\begin{array}{c}2 \\ 2\end{array}\right)=$ $\left(\begin{array}{c}s+2 \\ 3\end{array}\right)$ number of solutions. Similarly, for each pair $\left(t_{4}, t_{2}\right),\left(t_{4}, t_{1}\right),\left(t_{3}, t_{2}\right),\left(t_{3}, t_{1}\right)$ and $\left(t_{2}, t_{1}\right)$, we get $\left(\begin{array}{c}s+2 \\ 3\end{array}\right)$ linear syzygies. Note that the syzygies $x_{4} f_{t_{1}+1, t_{2}-1, t_{3}, t_{4}}-x_{3} f_{t_{1}, t_{2}, t_{3}, t_{4}}$ and $x_{3} f_{t_{1}, t_{2}, t_{3}, t_{4}}-x_{2} f_{t_{1}, t_{2}-1, t_{3}+1, t_{4}}$ give rise to another linear syzygy $x_{4} f_{t_{1}+1, t_{2}-1, t_{3}, t_{4}}-x_{2} f_{t_{1}, t_{2}-1, t_{3}+1, t_{4}}$. The same linear syzygy can also be obtained from a combination of linear syzygies that arise out of the pairs $\left(t_{1}, t_{4}\right)$ and $\left(t_{3}, t_{4}\right)$. Therefore, to get a minimal generating set, we only need to consider the linear syzygies corresponding to the pairs $\left(t_{1}, t_{2}\right),\left(t_{2}, t_{3}\right)$, and $\left(t_{3}, t_{4}\right)$. For each such pair, we have $\left(\begin{array}{c}s+2 \\ 3\end{array}\right)$ number of linear syzygies. Therefore, $\beta_{2}=3\left(\begin{array}{c}s+2 \\ 3\end{array}\right)$. 
Write the basis elements of $R^{3\left({ }^{(s+2}\right)}$ as

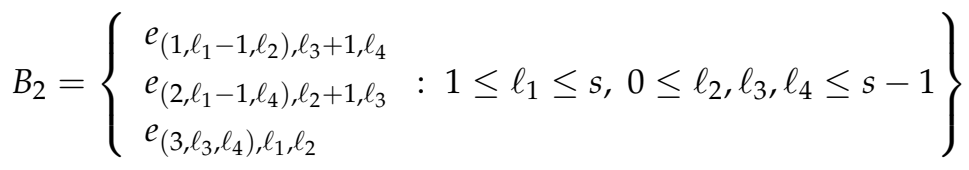

and define $\partial_{2}: R^{3\left(\begin{array}{c}s+1 \\ 2\end{array}\right)} \longrightarrow R^{\beta_{1}}$ by

$$
\begin{aligned}
\partial_{2}\left(e_{\left(1, \ell_{1}-1, \ell_{2}\right), \ell_{3}+1, \ell_{4}}\right) & =x_{2} e_{\ell_{1}-1, \ell_{2}, \ell_{3}+1, \ell_{4}}-x_{1} e_{\ell_{1}-1, \ell_{2}, \ell_{3}, \ell_{4}+1} ; \\
\partial_{2}\left(e_{\left(2, \ell_{1}-1, \ell_{4}\right), \ell_{2}+1, \ell_{3}}\right) & =x_{3} e_{\ell_{1}-1, \ell_{2}+1, \ell_{3}, \ell_{4}}-x_{2} e_{\ell_{1}-1, \ell_{2}, \ell_{3}+1, \ell_{4}} ; \\
\partial_{2}\left(e_{\left(3, \ell_{3}, \ell_{4}\right), \ell_{1}, \ell_{2}}\right) & =x_{4} e_{\ell_{1}, \ell_{2}, \ell_{3}, \ell_{4}}-x_{3} e_{\ell_{1}-1, \ell_{2}+1, \ell_{3}, \ell_{4}} .
\end{aligned}
$$

Now, we decipher the Betti numbers $\beta_{3}$ and $\beta_{4}$ to complete the resolution. The Hilbert series of $R / I^{S}$ is

$$
H\left(R / I^{s}, t\right)=\frac{\left(1-\beta_{1} t^{3 s}+\beta_{2} t^{3 s+1}-\beta_{3} t^{3 s+2}+\beta_{4} t^{3 s+3}\right)}{(1-t)^{4}}=\frac{p(t)}{(1-t)^{4}} .
$$

As $\operatorname{dim} R / I^{s}=2$, the polynomial $p(t)$ has a factor $(1-t)^{2}$. Note that $(1-t)^{2}$ is a monic polynomial of degree 2 , therefore we can write $p(t)=(1-t)^{2} \cdot q(t)$, where

$$
q(t)=\beta_{4} t^{3 s+1}+\left(2 \beta_{4}-\beta_{3}\right) t^{3 s}+a_{3 s-1} t^{3 s-1}+\cdots+a_{1} t+1 .
$$

On the other hand, we also have

$$
\begin{aligned}
\frac{p(t)}{(1-t)^{2}} & =\left(\sum_{n \geq 0}(n+1) t^{n}\right)\left(1-\beta_{1} t^{3 s}+\beta_{2} t^{3 s+1}-\beta_{3} t^{3 s+2}+\beta_{4} t^{3 s+3}\right) \\
& =\sum_{n=0}^{3 s-1}(n+1) t^{n}+\left(3 s+1-\beta_{1}\right) t^{3 s}+\left(3 s+2-2 \beta_{1}+\beta_{2}\right) t^{3 s+1}+\sum_{j \geq 3 s+2} a_{j} t^{j}
\end{aligned}
$$

For the expressions in Equations (5) and (6) to be equal, their respective coefficients should be equal. In particular, we should have that

$$
\beta_{4}=3 s+2-2 \beta_{1}+\beta_{2} \text { and } 2 \beta_{4}-\beta_{3}=3 s+1-\beta_{1} .
$$

On substituting $\beta_{1}=\left(\begin{array}{c}s+3 \\ 3\end{array}\right)$ and $\beta_{2}=3\left(\begin{array}{c}s+2 \\ 3\end{array}\right)$, we get $\beta_{4}=\left(\begin{array}{l}s \\ 3\end{array}\right)$ from the first equation and substituting the value in the second equation, we get $\beta_{3}=3\left(\begin{array}{c}s+1 \\ 3\end{array}\right)$. Now we verify that $a_{j}=0$ for all $j \geq 3 s+2$. Note that for $r \geq 2$, the coefficient of $t^{3 s+r}$ in Equation (6) is

$$
a_{3 s+r}=(3 s+r+1)+(r-2) \beta_{4}-(r-1) \beta_{3}+r \beta_{2}-(r+1) \beta_{1} .
$$

As $1-\beta_{1}+\beta_{2}-\beta_{3}+\beta_{4}=0$, this equation is reduced to $a_{3 s+r}=(3 s+3)-\beta_{3}+2 \beta_{2}-3 \beta_{1}$. Applying the binomial identify $\left(\begin{array}{c}n+1 \\ r+1\end{array}\right)=\left(\begin{array}{c}n \\ r+1\end{array}\right)+\left(\begin{array}{l}n \\ r\end{array}\right)$ repeatedly, we get $a_{3 s+r}=0$ for all $r \geq 2$. Hence the Hilbert series of $R / I^{s}$ is

$$
H\left(R / I^{s}, t\right)=\frac{1+2 t+3 t^{2}+4 t^{3}+\cdots+3 s t^{3 s-1}-\left(\left(\begin{array}{c}
s+3 \\
3
\end{array}\right)-3 s-1\right) t^{3 s}+\left(\begin{array}{c}
s \\
3
\end{array}\right) t^{3 s+1}}{(1-t)^{2}} .
$$

We now complete the description of the resolution. Write the basis elements of $R^{3\left({ }^{s+1}\right)}$ as

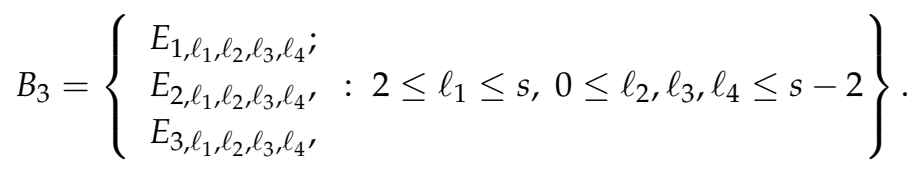


Note that $\left|B_{3}\right|=3\left(\begin{array}{c}s+1 \\ 3\end{array}\right)$. Now define the map $\partial_{3}: R^{3\left(\begin{array}{c}(+1 \\ 3\end{array}\right)} \longrightarrow R^{3\left({ }_{3}^{(+2}\right)}$ by

$$
\begin{aligned}
& \partial_{3}\left(E_{1, \ell_{1}, \ell_{2}, \ell_{3}, \ell_{4}}\right)=x_{4} e_{\left(2, \ell_{1}-1, \ell_{4}\right), \ell_{2}+1, \ell_{3}}+x_{4} e_{\left(1, \ell_{1}-1, \ell_{2}\right), \ell_{3}+1, \ell_{4}}-x_{3} e_{\left(3, \ell_{3}, \ell_{4}\right), \ell_{1}-1, \ell_{2}+1}
\end{aligned}
$$

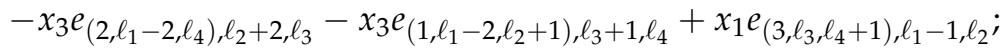

$$
\begin{aligned}
& \partial_{3}\left(E_{\left.2, \ell_{1}, \ell_{2}, \ell_{3}, \ell_{4}\right)}\right)=x_{4} e_{\left(1, \ell_{1}-1, \ell_{2}\right), \ell_{3}+1, \ell_{4}}-x_{3} e_{\left(1, \ell_{1}-2, \ell_{2}+1\right), \ell_{3}+1, \ell_{4}}-x_{2} e_{\left(3, \ell_{3}+1, \ell_{4}\right), \ell_{1}-1, \ell_{2}} \\
& +x_{1} e_{\left(3, \ell_{3}, \ell_{4}+1\right), \ell_{1}-1, \ell_{2} ;} \\
& \partial_{3}\left(E_{3, \ell_{1}, \ell_{2}, \ell_{3}, \ell_{4}}\right)=x_{3} e_{\left(1, \ell_{1}-2, \ell_{2}+1\right), \ell_{3}+1, \ell_{4}}-x_{2} e_{\left(2, \ell_{1}-2, \ell_{4}\right), \ell_{2}+1, \ell_{3}+1}-x_{2} e_{\left(1, \ell_{1}-2, \ell_{2}\right), \ell_{3}+2, \ell_{4}} \\
& +x_{1} e_{\left(2, \ell_{1}-2, \ell_{4}+1\right), \ell_{2}+1, \ell_{3}} \text {. }
\end{aligned}
$$

We now compute the kernel of $\partial_{3}$. Consider the set

$$
A=\left\{H_{\ell_{1}, \ell_{2}, \ell_{3}, \ell_{4}}: 3 \leq \ell_{1} \leq s, 0 \leq \ell_{2}, \ell_{3}, \ell_{4} \leq s-3\right\},
$$

where

$$
\begin{aligned}
H_{\ell_{1}, \ell_{2}, \ell_{3}, \ell_{4}}= & x_{4} E_{3, \ell_{1}, \ell_{2}, \ell_{3}, \ell_{4}}-x_{3} E_{2, \ell_{1}-1, \ell_{2}+1, \ell_{3}, \ell_{4}}-x_{3} E_{3, \ell_{1}-1, \ell_{2}+1, \ell_{3}, \ell_{4}}+x_{2} E_{1, \ell_{1}-1, \ell_{2}, \ell_{3}+1, \ell_{4}} \\
& -x_{1} E_{1, \ell_{1}-1, \ell_{2}, \ell_{3}, \ell_{4}+1}+x_{1} E_{2, \ell_{1}-1, \ell_{2}, \ell_{3}, \ell_{4}+1 .}
\end{aligned}
$$

It can be verified that $\partial_{3}\left(H_{\ell_{1}, \ell_{2}, \ell_{3}, \ell_{4}}\right)=0$, i.e., $A \subseteq \operatorname{ker} \partial_{3}$. Let $\ell_{1}^{\prime}=\ell_{1}-3$, then one has $\ell_{1}^{\prime}+\ell_{2}+$ $\ell_{3}+\ell_{4}=s-3$. The cardinality of $A$ is equal to the total number of non-negative integral solution of this linear equation, which is $\left(\begin{array}{l}s \\ 3\end{array}\right)$. As in the proof of Theorem 3, it can be seen that ker $\partial_{3}=\langle A\rangle$. Write the basis elements of $R^{(s)}$ as $B_{4}=\left\{G_{\ell_{1}, \ell_{2}, \ell_{3}, \ell_{4}}: 3 \leq \ell_{1} \leq s, 0 \leq \ell_{2}, \ell_{3}, \ell_{4} \leq s-3\right\}$ and define the $\operatorname{map} \partial_{4}: R^{\left(\begin{array}{c}s \\ 3\end{array}\right)} \longrightarrow R^{3\left(\begin{array}{c}(s+1 \\ 3\end{array}\right)}$ by

$$
\partial_{4}\left(G_{\ell_{1}, \ell_{2}, \ell_{3}, \ell_{4}}\right)=H_{\ell_{1}, \ell_{2}, \ell_{3}, \ell_{4}} .
$$

This is an injective map, and therefore we get the complete resolution:

$$
0 \rightarrow R(-3 s-3)^{\beta_{4}} \longrightarrow R(-3 s-2)^{\beta_{3}} \longrightarrow R(-3 s-1)^{\beta_{2}} \longrightarrow R(-3 s)^{\beta_{1}} \longrightarrow R .
$$

Note that in the above proof, we used $s \geq 3$ only to conclude that $\operatorname{pdim}\left(R / I^{s}\right)=4$. By Remark 4 , $\operatorname{depth}(R / I)=2$, and therefore $\operatorname{pdim}(R / I)=2$. Similarly, $\operatorname{depth}\left(R / I^{2}\right)=1$ and hence $\operatorname{pdim}\left(R / I^{2}\right)=$ 3. This forces $\partial_{2}$ to be injective when $s=1$ and $\partial_{3}$ to be injective when $s=2$. The computations of syzygies in the cases of resolution of $R / I$ and $R / I^{2}$ remain the same as given in the above proof. Therefore, we get resolutions truncated at $R^{\beta_{2}}$ in the case of $R / I$ and truncated at $R^{\beta_{3}}$ in the case of $R / I^{2}$, with the expressions for $\beta_{2}$ and $\beta_{3}$ coinciding with the ones given in the proof. Therefore, we can conclude that in this case, $\operatorname{reg}\left(I^{s}\right)=3 s$ for all $s \geq 1$.

As an immediate consequence, we obtain an expression for the asymptotic regularity of cover ideals of complete 4-partite graphs.

Theorem 6. Let $G$ denote a complete 4-partite graph with $V(G)=\sqcup_{i=1}^{4} V_{i}$ and $E(G)=\{\{a, b\}: a \in$ $\left.V_{i}, b \in V_{j}, i \neq j\right\}$. Set $V_{i}=\left\{x_{i 1}, \ldots, x_{i m_{i}}\right\}$ for $i=1, \ldots, 4$. Let $J_{G} \subset R=K\left[x_{i j}: 1 \leq i \leq 4 ; 1 \leq j \leq m_{i}\right]$ denote the cover ideal of $G$. Then the minimal free resolution of $R / J_{G}^{s}$ is of the form

$$
0 \rightarrow R^{\left(\begin{array}{c}
s \\
3
\end{array}\right)} \longrightarrow R^{3\left(\begin{array}{c}
(s+1 \\
3
\end{array}\right)} \longrightarrow R^{3\left(\begin{array}{c}
(s+2 \\
3
\end{array}\right)} \longrightarrow R^{\left(\begin{array}{c}
(s+3 \\
3
\end{array}\right)} \longrightarrow R .
$$


Set $\alpha=\left(s-\ell_{4}\right) m_{1}+\left(s-\ell_{3}\right) m_{2}+\left(s-\ell_{2}\right) m_{3}+\left(s-\ell_{1}\right) m_{4}$. Furthermore, we have

$$
\operatorname{reg}\left(J_{G}^{s}\right)=\max \begin{cases}\alpha, & \text { for } 0 \leq \ell_{1}, \ell_{2}, \ell_{3}, \ell_{4} \leq s, \\ \alpha+m_{4}-1, & \text { for } 1 \leq \ell_{1} \leq s, 0 \leq \ell_{2}, \ell_{3}, \ell_{4} \leq s-1, \\ \alpha+2 m_{4}-2, & \text { for } 2 \leq \ell_{1} \leq s, 0 \leq \ell_{2}, \ell_{3}, \ell_{4} \leq s-2, \\ \alpha+3 m_{4}-3, & \text { for } 3 \leq \ell_{1} \leq s, 0 \leq \ell_{2}, \ell_{3}, \ell_{4} \leq s-3,\end{cases}
$$

where $\ell_{1}+\ell_{2}+\ell_{3}+\ell_{4}=s$.

Proof. Let $X_{i}=\prod_{j=1}^{m_{i}} x_{i j}$. Then $J_{G}=\left(X_{1} X_{2} X_{3}, X_{1} X_{2} X_{4}, X_{1} X_{3} X_{4}, X_{2} X_{3} X_{4}\right)$. Then it follows from Theorem 5 that the minimal free resolution of $R / J_{G}^{s}$ is of the given form

$$
0 \rightarrow R^{\left(\begin{array}{c}
s \\
3
\end{array}\right)} \longrightarrow R^{3\left(\begin{array}{c}
(s+1 \\
3
\end{array}\right)} \longrightarrow R^{3\left(\begin{array}{c}
(s+2) \\
3
\end{array}\right)} \longrightarrow R^{\left(\begin{array}{c}
(s+3 \\
3
\end{array}\right)} \longrightarrow R \text {. }
$$

To compute the regularity of $R / J_{G}^{s}$, we first need to find the degree's of the generators of the syzygies. Following the notation of Theorem 5 , we have

$$
\begin{aligned}
& \operatorname{deg} \ell_{\ell_{1}, \ell_{2}, \ell_{3}, \ell_{4}}=\operatorname{deg}\left(X_{1}^{s-\ell_{4}} X_{2}^{s-\ell_{3}} X_{3}^{s-\ell_{2}} X_{4}^{s-\ell_{1}}\right)=\left(s-\ell_{4}\right) m_{1}+\left(s-\ell_{3}\right) m_{2}+\left(s-\ell_{2}\right) m_{3}+\left(s-\ell_{1}\right) m_{4}, \\
& \operatorname{deg} e_{\left(1, \ell_{1}-1, \ell_{2}\right), \ell_{3}, \ell_{4}}=\operatorname{deg} e_{\left(2, \ell_{1}-1, \ell_{4}\right), \ell_{2}+1, \ell_{3}}=\operatorname{deg} e_{\left(3, \ell_{3}, \ell_{4}\right), \ell_{1}, \ell_{2}}=\operatorname{deg} e_{\ell_{1}, \ell_{2}, \ell_{3}, \ell_{4}}+\operatorname{deg}\left(X_{4}\right) \text {, } \\
& \operatorname{deg} E_{1, \ell_{1}, \ell_{2}, \ell_{3}, \ell_{4}}=\operatorname{deg} E_{2, \ell_{1}, \ell_{2}, \ell_{3}, \ell_{4}}=\operatorname{deg} E_{3, \ell_{1}, \ell_{2}, \ell_{3}, \ell_{4}}=\operatorname{deg} e_{\ell_{1}, \ell_{2}, \ell_{3}, \ell_{4}}+2 \operatorname{deg}\left(X_{4}\right), \\
& \operatorname{deg} G_{\ell_{1}, \ell_{2}, \ell_{3}, \ell_{4}}=\operatorname{deg} e_{\ell_{1}, \ell_{2}, \ell_{3}, \ell_{4}}+3 \operatorname{deg}\left(X_{4}\right) \text {. }
\end{aligned}
$$

Therefore, by setting $\alpha=\operatorname{deg} \ell_{\ell_{1}, \ell_{2}, \ell_{3}, \ell_{4}}$, we get

$$
\operatorname{reg}\left(J_{G}^{s}\right)=\max \left\{\begin{array}{ll}
\alpha, & \text { for } 0 \leq \ell_{1}, \ell_{2}, \ell_{3}, \ell_{4} \leq s, \\
\alpha+\operatorname{deg}\left(X_{4}\right)-1, & \text { for } 1 \leq \ell_{1} \leq s, 0 \leq \ell_{2}, \ell_{3}, \ell_{4} \leq s-1, \\
\alpha+2 \operatorname{deg}\left(X_{4}\right)-2, & \text { for } 2 \leq \ell_{1} \leq s, 0 \leq \ell_{2}, \ell_{3}, \ell_{4} \leq s-2, \\
\alpha+3 \operatorname{deg}\left(X_{4}\right)-3, & \text { for } 3 \leq \ell_{1} \leq s, 0 \leq \ell_{2}, \ell_{3}, \ell_{4} \leq s-3,
\end{array},\right.
$$

where $\ell_{1}+\ell_{2}+\ell_{3}+\ell_{4}=s$.

Here also, we have obtained an expression for $\operatorname{reg}\left(J_{G}^{s}\right)$ not in the form of a linear polynomial. However, as we have demonstrated in the previous cases, this can always be derived for a given graph. Analyzing the interplay between the cardinalities of the partitions, one can obtain the polynomial expression. Let $m_{1}=m_{2}=m_{3}=m_{4}=m$. Then,

$$
\begin{aligned}
\alpha & =\left(s-\ell_{4}\right) m_{1}+\left(s-\ell_{3}\right) m_{2}+\left(s-\ell_{2}\right) m_{3}+\left(s-\ell_{1}\right) m_{4} \\
& =\left(4 s-\left(\ell_{1}+\ell_{2}+\ell_{3}+\ell_{4}\right)\right) m=3 m s
\end{aligned}
$$

Therefore $\operatorname{reg}\left(J_{G}^{s}\right)=3 m s+(3 m-3)$ for all $s \geq 3$.

Corollary 3. Let $m, r$ be any two positive integers. Consider the arithmetic progression $m_{1}=m, m_{2}=m+r$, $m_{3}=m+2 r$, and $m_{4}=m+3 r$ in Theorem 6 . Then, for all $s \geq 3$, we have

$$
\operatorname{reg}\left(J_{G}^{s}\right)=s(3 m+6 r)+3 m-3 .
$$

Proof. We have from Theorem $6, \alpha=\left(s-\ell_{4}\right) m_{1}+\left(s-\ell_{3}\right) m_{2}+\left(s-\ell_{2}\right) m_{3}+\left(s-\ell_{1}\right) m_{4}$. On substituting the values of $m_{i}$ 's in $\alpha$, we get

$$
\alpha=s(4 m+6 r)-m \ell_{4}-(m+r) \ell_{3}-(m+2 r) \ell_{2}-(m+3 r) \ell_{1}
$$


By Theorem 6, we have for all $s \geq 3$,

$$
\operatorname{reg}\left(J_{G}^{s}\right)=\max \begin{cases}\alpha, & \text { for } 0 \leq \ell_{1}, \ell_{2}, \ell_{3}, \ell_{4} \leq s \\ \alpha+(m+3 r)-1, & \text { for } 1 \leq \ell_{1} \leq s, 0 \leq \ell_{2}, \ell_{3}, \ell_{4} \leq s-1, \\ \alpha+2(m+3 r)-2, & \text { for } 2 \leq \ell_{1} \leq s, 0 \leq \ell_{2}, \ell_{3}, \ell_{4} \leq s-2, \\ \alpha+3(m+3 r)-3, & \text { for } 3 \leq \ell_{1} \leq s, 0 \leq \ell_{2}, \ell_{3}, \ell_{4} \leq s-3\end{cases}
$$

where $\ell_{1}+\ell_{2}+\ell_{3}+\ell_{4}=s$. To achieve the maximum value of $\alpha$, negative terms in $\alpha$ should be minimum. The coefficient of $\ell_{1}$ in negative terms in $\alpha$ is largest, so $\ell_{1}$ should be assigned the minimum value. After assigning the minimum value to $\ell_{1}$, assign the minimum value to $\ell_{2}$, and, similarly, the minimum value to $\ell_{3}$. Then assign $\ell_{4}=s-\ell_{1}-\ell_{2}-\ell_{3}$. For instance, to get the maximum of $\alpha$ when $1 \leq \ell_{1} \leq s, 0 \leq \ell_{2}, \ell_{3}, \ell_{4} \leq s-1$, put $\ell_{1}=1, \ell_{2}=0, \ell_{3}=0$, and $\ell_{4}=s-1$. With appropriate substitution, we get for all $s \geq 3$

$$
\operatorname{reg}\left(J_{G}^{s}\right)=\max \begin{cases}s(3 m+6 r), & \text { for } 0 \leq \ell_{1}, \ell_{2}, \ell_{3}, \ell_{4} \leq s, \\ s(3 m+6 r)+m-1, & \text { for } 1 \leq \ell_{1} \leq s, 0 \leq \ell_{2}, \ell_{3}, \ell_{4} \leq s-1, \\ s(3 m+6 r)+2 m-2, & \text { for } 2 \leq \ell_{1} \leq s, 0 \leq \ell_{2}, \ell_{3}, \ell_{4} \leq s-2, \\ s(3 m+6 r)+3 m-3, & \text { for } 3 \leq \ell_{1} \leq s, 0 \leq \ell_{2}, \ell_{3}, \ell_{4} \leq s-3 .\end{cases}
$$

Clearly for all $m \geq 1$, and for all $s \geq 3$, we get

$$
\operatorname{reg}\left(J_{G}^{s}\right)=s(3 m+6 r)+3 m-3
$$

\subsection{Complete m-Partite Graphs}

Let $G$ be a complete graph on $m$-vertices. Then the cover ideal $J_{G}$ of $G$ is generated by $\left\{x_{1} \cdots \hat{x}_{i} \cdots x_{m}: 1 \leq i \leq m\right\}$. It follows from Remark 4 that depth $R / J_{G}^{s}=0$ for all $s \geq m-1$. Moreover, by Remark 3, we know that $R / J_{G}^{s}$ has linear resolution for all $s \geq 1$. Therefore, the minimal graded free resolution of $R / J_{G}^{s}$ for all $s \geq m-1$ is of the form

$$
0 \rightarrow R(-s(m-1)-m+1)^{\beta_{m}} \longrightarrow \rightarrow \cdots \rightarrow R(-s(m-1)-1)^{\beta_{2}} \longrightarrow R(-s(m-1))^{\beta_{1}} \longrightarrow R \text {. }
$$

Let $g_{1}, g_{2}, \ldots, g_{m}$ be the minimal generators $J_{G}$. Then, the elements in $J_{G}^{s}$ consist of elements $T_{\ell_{1}, \ell_{2}, \ldots, \ell_{m}}=g_{1}^{\ell_{1}} g_{2}^{\ell_{2}} \ldots g_{m}^{\ell_{m}}$, such that $\ell_{1}+\ell_{2}+\cdots+\ell_{m}=s$ and $0 \leq \ell_{i} \leq s$. Therefore the total number of elements in $J_{G}^{s}$ is same as the total number of non-negative integral solution to the linear equation $\ell_{1}+\ell_{2}+\cdots+\ell_{m}=s$, which is $\left(\begin{array}{c}s+m-1 \\ m-1\end{array}\right)$. Thus, $\mu\left(J_{G}^{s}\right)=\left(\begin{array}{c}s+m-1 \\ m-1\end{array}\right)$. Therefore, $\beta_{1}=\left(\begin{array}{c}s+m-1 \\ m-1\end{array}\right)$.

Let $\left\{e_{\ell_{1}, \ell_{2}, \ldots, \ell_{m}} \mid 0 \leq \ell_{i} \leq s\right.$; and $\left.\ell_{1}+\ell_{2}+\cdots+\ell_{m}=s\right\}$ denote the standard basis for $R^{\beta_{1}}$. Let $\partial_{1}: R^{\beta_{1}} \longrightarrow R$ be the map $\partial_{1}\left(e_{\ell_{1}, \ell_{2}, \ldots, \ell_{m}}\right)=T_{\ell_{1}, \ell_{2}, \ldots, \ell_{m}}$. As done in the proofs of Theorems 3 and 5 , we can see that the first syzygy is given by the relations of the form

$$
x_{i} \cdot T_{\ell_{1}, \ell_{2}, \cdots, \ell_{i-1}, \ell_{i}-1, \ell_{i+1}+1, \cdots, \ell_{m}}-x_{i+1} \cdot T_{\ell_{1}, \ell_{2}, \cdots, \ell_{i-1}, \ell_{i}, \ell_{i+1}, \cdots, \ell_{m}}=0
$$

for each $1 \leq i \leq m-1$. Set $\ell_{i}-1=\ell_{i}^{\prime}$ and $\ell_{i+1}+1=\ell_{i+1}^{\prime}$. Then it can be seen that, for each $1 \leq i \leq m-1$, there exist as many such relations as the number of non-negative integer solutions of $\ell_{1}+\cdots+\ell_{i}^{\prime}+\cdots \ell_{m}=s-1$. Therefore, the total number of such linear relations is $(m-1)\left(\begin{array}{c}s+m-2 \\ m-1\end{array}\right)$. Therefore $\beta_{2}=\left(\begin{array}{c}m-1 \\ 1\end{array}\right)\left(\begin{array}{c}s+m-2 \\ m-1\end{array}\right)$. However it is not very difficult to realize that writing down the higher syzygy relations are quite challenging. Based on Theorems 3 and 5 and some of the experimental results using the computational commutative algebra package Macaulay 2 [17], we propose the following conjecture. 
Conjecture 1. Let $R=K\left[x_{1}, x_{2}, \ldots, x_{m}\right]$ and let $J$ be the cover ideal of the complete graph $K_{m}$. The minimal graded free resolution of $R / I^{s}$ for all $s \geq m-1$ is of the form

$$
0 \rightarrow R(-s(m-1)-m+1)^{\beta_{m}} \longrightarrow \cdots \longrightarrow R(-s(m-1)-1)^{\beta_{2}} \longrightarrow R(-s(m-1))^{\beta_{1}} \longrightarrow R,
$$

where

$$
\beta_{i}=\left(\begin{array}{c}
m-1 \\
i-1
\end{array}\right)\left(\begin{array}{c}
s+m-i \\
m-1
\end{array}\right)
$$

Notice that proving the above conjecture will give the Betti numbers of powers of cover ideals of complete $m$-partite graphs. We conclude our article by proposing an expression for the regularity of powers of the cover ideals of complete $m$-partite graphs:

Conjecture 2. Let $G$ denote a complete m-partite graph with $V(G)=\sqcup_{i=1}^{m} V_{i}$ and $E(G)=\{\{a, b\}: a \in$ $\left.V_{i}, b \in V_{j}, i \neq j\right\}$. Set $V_{i}=\left\{x_{i 1}, \ldots, x_{i n_{i}}\right\}$ for $i=1, \ldots, m$. Let $J_{G} \subset R=K\left[x_{i j}: 1 \leq i \leq m ; 1 \leq j \leq n_{i}\right]$ denote the cover ideal of $G$. Let $0 \leq \ell_{1}, \ell_{2}, \ldots, \ell_{m} \leq s$ be integers such that $\ell_{1}+\ell_{2}+\cdots+\ell_{m}=s$. Set

$$
\alpha=s \cdot\left(\sum_{i=1}^{m} n_{i}\right)-\sum_{i=1}^{m} n_{i} \ell_{m+1-i} .
$$

Then for all $s \geq m-1$, one has

$$
\operatorname{reg}\left(J_{G}^{s}\right)=\max \begin{cases}\alpha, & \text { for } 0 \leq \ell_{1}, \ell_{2}, \cdots, \ell_{m} \leq s, \\ \alpha+n_{m}-1, & \text { for } 1 \leq \ell_{1} \leq s, 0 \leq \ell_{2}, \cdots, \ell_{m} \leq s-1, \\ \alpha+2\left(n_{m}-1\right), & \text { for } 2 \leq \ell_{1} \leq s, 0 \leq \ell_{2}, \cdots, \ell_{m} \leq s-2, \\ & \vdots \\ \alpha+(m-1)\left(n_{m}-1\right), & \text { for } m-1 \leq \ell_{1} \leq s, 0 \leq \ell_{2}, \cdots, \ell_{m} \leq s-(m-1) .\end{cases}
$$

Author Contributions: The results here are the outcome of several discussions that we held together. The authors contributed to this work equally.

Funding: This research received no external funding.

Acknowledgments: Part of the work was done while the second author was visiting Indian Institute of Technology Madras. He would like to thank IIT Madras for their hospitality during the visit. All our computations were done using Macaulay 2. We would like to thank Huy Tài Hà and S. A. Seyed Fakhari for their comments which helped us improve the exposition.

Conflicts of Interest: The authors declare no conflict of interest.

\section{References}

1. Peeva, I.; Stillman, M. The minimal free resolution of a Borel ideal. Expo. Math. 2008, 26, 237-247. [CrossRef]

2. Buchsbaum, D.A.; Eisenbud, D. Generic free resolutions and a family of generically perfect ideals. Adv. Math. 1975, 18, 245-301. [CrossRef]

3. Guardo, E.; Van Tuyl, A. Powers of complete intersections: graded Betti numbers and applications. Ill. J. Math. 2005, 49, 265-279. [CrossRef]

4. Conca, A. Hilbert function and resolution of the powers of the ideal of the rational normal curve. J. Pure Appl. Algebra 2000, 152, 65-74. [CrossRef]

5. Herzog, J.; Hibi, T.; Zheng, X. Monomial ideals whose powers have a linear resolution. Math. Scand. 2004, 95, 23-32. [CrossRef]

6. Conca, A.; Herzog, J. Castelnuovo-Mumford regularity of products of ideals. Collect. Math. 2003, 54, 137-152.

7. Ene, V.; Olteanu, A. Powers of lexsegment ideals with linear resolution. Ill. J. Math. 2012, 56, 533-549. [CrossRef] 
8. Kodiyalam, V. Asymptotic behaviour of Castelnuovo-Mumford regularity. Proc. Amer. Math. Soc. 2000, 128, 407-411. [CrossRef]

9. Cutkosky, S.D.; Herzog, J.; Trung, N. Asymptotic behaviour of the Castelnuovo-Mumford regularity. Compos. Math. 1999, 118, 243-261. [CrossRef]

10. Seyed Fakhari, S.A. Symbolic powers of cover ideal of very well-covered and bipartite graphs. arXiv 2016, arXiv:1604.0065.

11. Hang, N.T.; Trung, T.N. Regularity of powers of cover ideals of unimodular hypergraphs. J. Algebra 2018, 513, 159-176. [CrossRef]

12. Mohammadi, F.; Moradi, S. Weakly polymatroidal ideals with applications to vertex cover ideals. Osaka J. Math. 2010, 47, 627-636.

13. Morey, S. Depths of powers of the edge ideal of a tree. Comm. Algebra 2010, 38, 4042-4055. [CrossRef]

14. Herzog, J.; Hibi, T. The depth of powers of an ideal. J. Algebra 2005, 291, 534-550. [CrossRef]

15. Herzog, J.; Hibi, T. Monomial Ideals, Volume 260 of Graduate Texts in Mathematics; Springer: London, UK, 2011.

16. Chen, J.; Morey, S.; Sung, A. The stable set of associated primes of the ideal of a graph. Rocky Mt. J. Math. 2002, 32, 71-89. [CrossRef]

17. Grayson, D.R.; Stillman, M.E. Macaulay2, A Software System for Research in Algebraic Geometry. Available online: http:/ / www.math.uiuc.edu/Macaulay2/ (accessed on 17 February 2018).

(c) 2019 by the authors. Licensee MDPI, Basel, Switzerland. This article is an open access article distributed under the terms and conditions of the Creative Commons Attribution (CC BY) license (http://creativecommons.org/licenses/by/4.0/). 